\title{
Size dependence in chord characteristics from simulated and observed continental shallow cumulus
}

\author{
Philipp J. Griewank ${ }^{1}$, Thijs Heus ${ }^{2}$, Neil P. Lareau ${ }^{3}$, and Roel A. J. Neggers ${ }^{1}$ \\ ${ }^{1}$ University of Cologne, Cologne, Germany \\ ${ }^{2}$ Cleveland State University, Cleveland, Ohio, USA \\ ${ }^{3}$ University of Nevada, Reno, Nevada, USA
}

Correspondence: Philipp J. Griewank (philipp.griewank@uni-koeln.de)

Received: 8 April 2020 - Discussion started: 29 April 2020

Revised: 6 July 2020 - Accepted: 18 July 2020 - Published: 3 September 2020

\begin{abstract}
In this study we compare long-term Doppler and Raman lidar observations against a full month of large eddy simulations of continental shallow cumulus clouds. The goal is to evaluate if the simulations can reproduce the mean observed vertical velocity and moisture structure of cumulus clouds and their associated subcloud circulations, as well as to establish if these properties depend on the size of the cloud. We propose methods to compare continuous chords of cloud detected from Doppler and Raman lidars with equivalent chords derived from 1D and 3D model output. While the individual chords are highly variable, composites of thousands of observed and millions of simulated chords contain a clear signal. We find that the simulations underestimate cloud size and fraction but successfully reproduce the observed structure of vertical velocity and moisture perturbations. There is a clear scaling of vertical velocity and moisture anomalies below the chords with chord size, but the moisture anomalies are only 1\%-2\% higher than the horizontal mean values. The differences between the observations and simulations are smaller than the difference in sampling the modeled chords in time or space. The shape of the vertical velocity and moisture anomalies from cloud chords sampled spatially from 3D model snapshots is almost perfectly symmetric. In contrast, the chords sampled temporally from the lidar observations and $1 \mathrm{D}$ model output have a marked asymmetry, with stronger updrafts and higher moisture anomalies occurring earlier on.
\end{abstract}

\section{Introduction}

Shallow cumulus cloud populations contain a wide range of spatial, temporal, and physical-dynamical variability. The individual clouds themselves vary greatly in size, lifetime, depth, and microphysical properties. But even statistics of large shallow cumulus populations differ as well, with submesoscale dynamics, such as cold pools, causing large-scale organization and aggregation (Sengupta et al., 1990; Nair et al., 1998). This high individual and statistical variability, combined with their comparatively small temporal and spatial scales, make shallow cumulus a tricky aspect of the atmosphere to represent numerically in weather and climate models (e.g., Neggers and Siebesma, 2013; Nam et al., 2014), from which they cannot be neglected due to their critical contribution to Earth's energy and water budget (e.g., Sherwood et al., 2014; Bony et al., 2015; Brient and Schneider, 2016).

One useful paradigm that simplifies representing the effects of sub-grid-scale shallow cumulus on resolved-scale flow is the assumption that various properties of shallow cumulus are size-dependent, which enables the representation of the high variability in cumulus clouds through a limited number of shallow convective plumes of differing size (e.g., Arakawa and Schubert, 1974; Neggers, 2015; Olson et al., 2019). The idea that larger shallow cumulus clouds have stronger updrafts than small shallow cumulus clouds is as intuitive as it is old (e.g., Plank, 1969; Raga et al., 1990; Benner and Curry, 1998; Zhao and Girolamo, 2007; Yuan, 2011). Assuming shallow cumulus clouds are created by buoyant plumes that are slowed via entrainment with the dry surrounding air (Turner, 1962; Simpson and Wiggert, 1969; Warner, 1970a), it follows that larger plumes could 
rise faster by either being more buoyant, entraining less, or a mix of both. The assumed size dependence of plume entrainment is a cornerstone of the first bin-macrophysics approach in spectral modeling introduced by Arakawa and Schubert (1974). Model studies support this entrainment dependence (e.g., Zhao and Austin, 2005; Peters et al., 2020), and the dependence is still being used today (e.g., Neggers, 2015; Olson et al., 2019).

The potential use of size dependence in convective parameterization has motivated historic and recent efforts to observe the existence of this dependence in nature, but detecting this dependence in observations has proven to be quite difficult. This is in part because due to the relatively small size of shallow cumulus clouds, which predominantly have a horizontal area below a single square kilometer, the resolution of 3D scanning radars is insufficient to fully resolve the associated wind field. The short life span and nonstationary character further complicate measurements, as only a short time window exists to measure the active updraft. Airplanes can fly right through the center of cumulus clouds to measure a 1D track though the cloud and have a long and successful history of studying the structure of cumulus clouds and their subsiding shells, especially for maritime cumulus (e.g., Warner, 1970b; French et al., 1999; Laird et al., 2000; Rodts et al., 2003; Neggers et al., 2006; Heus and Jonker, 2008; Wang and Geerts, 2010, 2011). However, the high operating costs of aircraft mean that for practical reasons the total number of clouds that can be sampled via aircraft is limited, and commonly only a 1D line is sampled along a specific height. Other novel approaches such as tethered balloons (Kitchen and Caughey, 2007), helicopters (Siebert et al., 2006), and radar chaff detected from a plane-mounted radar (Jung and Albrecht, 2014) suffer from similar practical constraints. Given the large role turbulence and individual thermals play in shallow cumulus development, many samples are needed to detect if a size dependence is present.

This is where upward-facing lidar observations excel. After years of steady deployment of Doppler lidars at the ARMSGP (Atmospheric Radiation Measurement-Southern Great Plains) and other super-sites, thousands of shallow cumulus clouds have been measured as they pass over lidars. While the Doppler lidars cannot measure above the boundary layer or inside the cloud, they have sufficiently high temporal and spatial resolution to resolve the vertical velocity below shallow cumulus clouds. Both Lamer and Kollias (2015) and Lareau et al. (2018) successfully collected over 1000 Doppler lidar observations of shallow cumulus clouds at the ARMSGP site, showing that updraft strength increases with cloud chord length. In regards to the spatial structure, Lareau et al. (2018) show that on average a clear 2D updraft exists below the cloud, and Lareau (2020) achieved a similar result using Raman lidar measurements of water vapor.

In contrast to observations, large eddy simulation (LES) runs of shallow cumulus in principle allow for unlimited sampling of all simulated properties in $4 \mathrm{D}$. Studies such as
Dawe and Austin (2012), Böing et al. (2012), and Neggers (2015) have taken advantage of this fact to study the cloud properties of maritime cumulus in an individual LES. However, LES models still represent a simplification of reality and should be critically compared to measurements where possible. But bringing together LES experiments and observations on cumulus statistics, in particular detailed dependencies on size, requires large numbers of both observations and LES experiments. This is especially true for continental shallow cumulus clouds that have a marked daily cycle and strong day-to-day variability. But thanks to ongoing efforts to run semicontinuous LES experiments for observational super-sites in Europe (Neggers et al., 2012; Schalkwijk et al., 2015; van Laar et al., 2019) and the US (LASSO - LES ARM Symbiotic Simulation and Observation; Gustafson et al., 2018, 2020), we now have the ability to robustly compare observed and simulated cloud statistics, as done recently for cloud-base vertical velocity by Endo et al. (2019). Maritime cumulus comparisons require fewer simulations, as shown by the analysis of cloud-base mass flux at the Barbados Cloud Observatory by Sakradzija and Klingebiel (2020).

In this study we take advantage of the newly available large numbers of high-resolution simulations and long-term lidar observations. We do so by linking the observational methods of Lareau et al. (2018) and Lareau (2020) with the LASSO strategy of running LES experiments of all shallow cumulus days from 2015 to 2018 at the ARM-SGP site (Gustafson et al., 2018, 2020). In order to get a resolution of $25 \mathrm{~m}$, which is higher than the default LASSO settings, we use the LASSO forcing data to simulate 28 cumulus days with the MicroHH model (van Heerwaarden et al., 2017).

The main goals of this paper are (1) to determine the size sensitivity of subcloud vertical velocity and moisture anomaly fields and (2) to determine if the MicroHH simulations provide a reliable approximation of shallow cumulus cloud statistics. The goals are accomplished by making a one-to-one comparison of observed and simulated cloud chords and their subcloud circulation. To achieve this oneto-one comparison we sample chords from 1D column output, which captures the temporal evolution of clouds as they pass over a fixed point, and from 3D model output snapshots, which are frozen in time. After accomplishing the main goals of this paper we use our large data set to quantify the sampling uncertainty of various observed chord properties as a function of lidar deployment days.

This paper begins with an explanation of the data and simulations, followed by a comparison of simulated and observed cloud fraction and cloud-base height at the ARM-SGP site in Sect. 2. We then detail the methods for extracting chords from 1D (Sect. 3.1) and 3D (Sect. 3.2) model output to compare against the lidar observations. Before we evaluate the scale dependence and shape of vertical velocity and moisture anomalies in Sects. 5 and 6, we first compare the observed and simulated chord distributions in Sect. 4 to test 
how representative the simulated chords are and how much our results depend on the precise chord definitions used. We end our results with an analysis of the sampling uncertainty in Sect. 7 in which we quantify how the uncertainty decreases for various variables when more days of lidar observations are available. We end with a conclusion and discussion in Sect. 8 .

\section{Observations and simulations}

\subsection{Observations}

In this subsection we provide a brief overview of the instrumentation used to observe the vertical velocity and water vapor mixing ratio. For a more in-depth description of the data and how they were post-processed we refer the reader to Lareau (2020) (vertical velocity) and Lareau (2020) (water vapor mixing ratio).

\subsubsection{Vertical velocity}

The vertical velocity from the surface up to the cloud base is observed with a network of five Doppler lidars (DLs) located at ARM-SGP (Newsom, 2010). The lidar network is arranged with four outlying sites (E32, E37, E39, E41) and one central location (C1). The outlying sites fall on an approximately $50 \mathrm{~km}$ radius circle centered on $\mathrm{C} 1$. The DLs are active ground-based infrared $(1.5 \mu \mathrm{m})$ laser remote sensors that provide range- and time-resolved profiles of the line-ofsight velocity (i.e., the vertical velocity when pointed vertically) and the attenuated backscatter coefficient (hereafter backscatter) over a range of 9-12 km from the surface (depending on the lidar and its settings). The lidar data are processed at $30 \mathrm{~m}$ range gate resolution and $1.3 \mathrm{~s}$ temporal resolution. The DL is sensitive to micron-scale aerosol, which provides a tracer for boundary layer flows. As such the lidars provide an ideal tool for resolving the time-height structure of large convective eddies in the boundary layer. The lidar beams also rapidly attenuate in liquid water, thereby enabling cloud-base detection. The DL is also used to identify the time-varying convective boundary layer (CBL) height based on a threshold of the vertical velocity variance computed for 15 min intervals (Tucker et al., 2009). Here we use a variance threshold of $0.08 \mathrm{~m}^{2} \mathrm{~s}^{-2}$, which Lareau et al. (2018) show produces a good representation of CBL heights during shallow cumulus $(\mathrm{ShCu})$ conditions.

\subsubsection{Water vapor mixing ratio}

The subcloud and cloud-base water vapor mixing ratio $(q)$ is determined using an ultraviolet (UV) Raman lidar (RL), which is located adjacent to the DL at C1. The RL is sensitive to both molecular and aerosol backscatter, with the molecular backscatter used in the retrieval of the water vapor mixing ratio (Wulfmeyer et al., 2010). The retrieved wa- ter vapor profiles are available at $10 \mathrm{~s}$ temporal and $50 \mathrm{~m}$ spatial resolution (Raman lidar vertical profiles, 10SRLPROFMR1TURN). The ability of RL to measure the first through third moments of the boundary layer water vapor mixing ratio is well-established (Wulfmeyer et al., 2010; Turner et al., 2014a), and the RL at ARM-SGP has been validated against aircraft data (Turner et al., 2014b).

\subsection{Model}

We are basing our analysis on cloud fields generated with $\mathrm{Mi}$ croHH (van Heerwaarden et al., 2017). This large eddy simulation model has been validated against a wide range of standard cases, including shallow cumulus intercomparison cases in marine (e.g., BOMEX; Siebesma et al., 2003) and continental (e.g., ARM; Brown et al., 2002) conditions. For the current study, we simulated $28 \mathrm{~d}$ with shallow cumulus convection over the Department of Energy's Atmospheric Radiation Measurement site in the Southern Great Plains (ARMSGP) based on the Large-Eddy Simulation (LES) ARM Symbiotic Simulation and Observation (LASSO; Gustafson et al., 2017) database. These realistic and routine simulations of cumulus fields over the ARM-Southern Great Plains observatory in Oklahoma are run using a variety of initial conditions and model settings. For each day in the list in Table 1 , we selected the configurations with the best match to the observations in cloud cover and liquid water path. We ran one simulation for each day in the LASSO version 1 release, as long as the cloud cover and liquid water path have some skill in the best-matching simulation according to the LASSO Bundle Browser (https://adc.arm.gov/lassobrowser, last access: 26 August 2020) (skill scores above 0.3). Since the simulations in the LASSO database were done on a relatively coarse resolution of $100 \mathrm{~m}$ and with insufficient temporal output frequency to mimic lidar observations, we reran all cases with $\mathrm{MicroHH}$ on a $25 \mathrm{~m}$ resolution in all directions and a horizontal domain size of $25.6 \times 25.6 \mathrm{~km}^{2}$. Between a height of $6 \mathrm{~km}$ to the domain top at $9 \mathrm{~km}$, the vertical grid stretches from 25 to $150 \mathrm{~m}$. Adaptive time stepping with a constant Courant-Friedrichs-Lewy criterion results in a time step typically between 1 and $2 \mathrm{~s}$. The simulations were run with periodic boundary conditions, homogeneous and prescribed surface fluxes, and a prescribed radiative tendency profile. Two-moment warm microphysics was used, even though precipitation was negligible. Average statistics and 3D output of all thermodynamic variables were provided every $1800 \mathrm{~s}$. Raw column data were provided every time step (i.e., about every second) at $4^{2}=16$ locations throughout the domain, with each column spaced $6.4 \mathrm{~km}$ apart in both the $x$ and $y$ direction.

\subsection{Simulation evaluation}

Before beginning our analysis of cloud chords we first determine to what degree our simulated cloud-topped bound- 
Table 1. Dates of simulations included in the analysis (month/day).

\begin{tabular}{lllllll}
\hline 2015 & $06 / 06$ & $06 / 09$ & $06 / 27$ & $08 / 01$ & & \\
2016 & $05 / 18$ & $05 / 30$ & $06 / 10$ & $06 / 11$ & $06 / 19$ & $06 / 25$ \\
& $07 / 19$ & $07 / 20$ & $08 / 18$ & $08 / 19$ & $08 / 30$ & \\
2017 & $05 / 09$ & $06 / 05$ & $06 / 27$ & $07 / 04$ & $07 / 16$ & $07 / 19$ \\
& $07 / 20$ & $07 / 22$ & & & & \\
2018 & $05 / 22$ & $06 / 06$ & $07 / 05$ & $07 / 09$ & $07 / 10$ & \\
\hline
\end{tabular}

ary layer matches the observations by comparing cloud-base height and cloud fraction. Thanks to the evaluation data provided as part of the LASSO library along with the forcing data, we can easily compare the hourly cloud-base height and cloud fraction. We find that the cloud-base heights of simulations and observations agree well, with the average simulated cloud base being only $35 \mathrm{~m}$ lower than the observed (Fig. 1). As expected, the boundary layer deepening over the course of the day is clearly visible in the individual simulations. This good agreement of cloud-base height matches was also found for the original LASSO simulations (Gustafson et al., 2020) and can be directly attributed to the forcing data provided by the LASSO project being well-calibrated.

In contrast to cloud-base height, the modeled and observed cloud fractions align less well (Fig. 2). The observed hourly cloud fraction has a higher temporal variability, with measurement-to-measurement changes of up to 0.5 (e.g., 20170716; Fig. 2). Such strong shifts likely represent the sampling bias in a spatially and temporally heterogeneous cumulus-topped boundary layer (Rossow, 1989). Since the cloud fraction from the MicroHH simulations is calculated from 3D snapshots of the full $25.6 \times 25.6 \mathrm{~km}^{2}$ model domain, the cloud fraction is captured much more robustly, leading to a smoother daily cycle.

The two observational products provided by the LASSO library are the Total Sky Imager (TSI) cloud fraction and the low cloud fraction provided by the Active Remote Sensing of Clouds (ARSCL) value-added product. These two products differ by roughly 0.2 at any moment, and when averaging over all $24 \mathrm{~d}$ a mean difference of roughly 0.1 remains. But the observations are clear enough to show that the MicroHH simulations are substantially underestimating cloud fractions in two cases. The first is when high cloud fractions occur before noon, for example for days 20160818 and 20170627. We attribute this to the presence of non-convective clouds. Non-convective clouds should be screened out in our analysis as detailed in Sect. 3, so we do not expect the lack of these early clouds in the LES to affect our analysis. The second clear case of cloud underestimation in the simulations occurs on three consecutive days in 2017 when, for reasons we do not know, the cloud fraction remains below 0.1 throughout the day (20170719, 20170720, 20170721; Fig. 2).

In regards to the mean cloud fraction over all available data points after 09:00 (North American Central Time), the simulations underestimate cloud fraction by $15 \%$ compared to

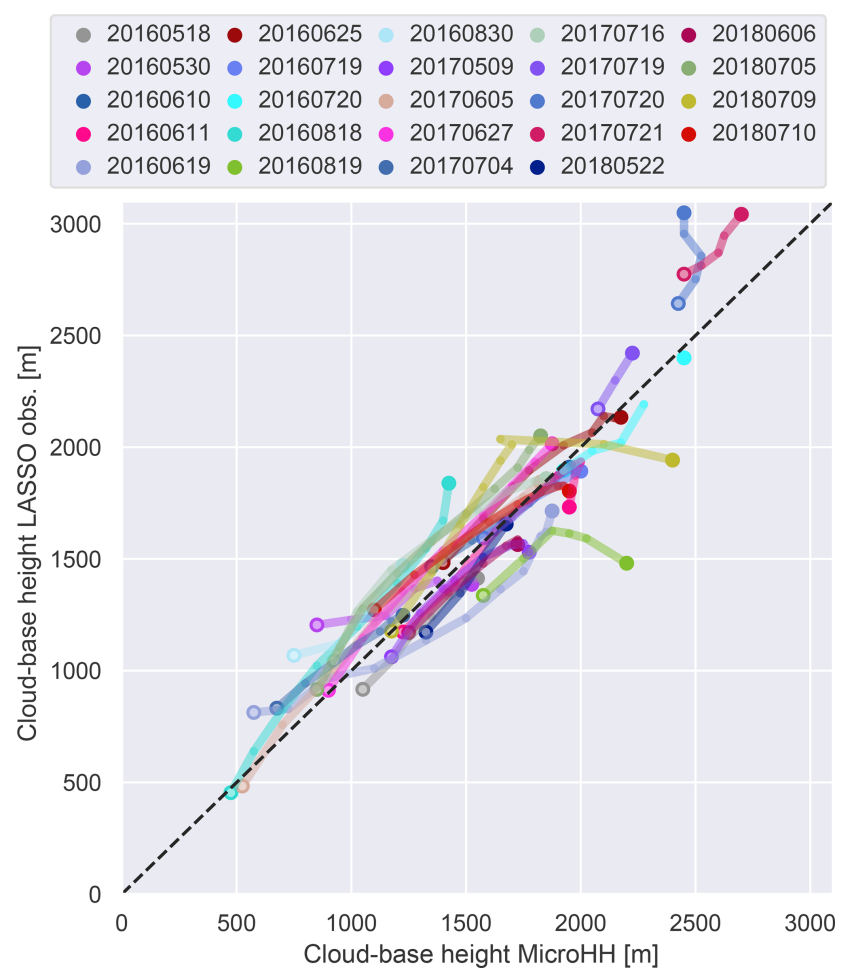

Figure 1. Cloud-base height of the MicroHH simulations compared to the cloud-base height provided by the LASSO Bundle Browser, which is an ARSCL value-added data product. Points are only shown when both the model and observations have a well-defined cloud base at the same time, and the observations are only used when the quality flag is good. Empty circles mark the earliest time point and the large dots the latest.

the TSI cloud fraction and by $60 \%$ compared to the ARSCL value-added product. This general underestimation is also present in the original LASSO simulations as shown by Gustafson et al. (2020) and matches the experience of previous continental shallow cumulus LES studies over the ARM-SGP site (Zhang et al., 2017) and over Cabauw in the Netherlands (Schalkwijk et al., 2015). Recently, Fast et al. (2019) showed that using a more realistic surface moisture distribution leads to larger and longer-lived clouds, indicating that our cloud underestimation could be due to the homogeneous surface conditions of the LASSO setup. According to Gustafson et al. (2020), the simulated clouds in the LASSO simulations appear roughly $2 \mathrm{~h}$ later than in the observations, which could contribute to why on average the MicroHH cloud underestimation is stronger before 15:00 (bottom left plot in Fig. 2). For the rest of the paper we will work with the assumption that the simulated cloud-base height is accurate and that the simulated cloud fraction is too low by an unknown amount between $10 \%$ and $60 \%$. 


\section{Detecting cloud chords and interpolating scenes}

To avoid confusion we will first explain how we use the terms cloud chord, chord-base height, and scene in this paper as illustrated in Fig. 3. By cloud chord we mean a continuous 1D string of cloud. Cloud chords are also referred to as traverses (e.g., Warner, 1970b) or intercepts (e.g., Rodts et al., 2003) in airplane studies and can theoretically fully describe the cloud field if one assumes a simplified cloud shape (Wood and Field, 2011). As we are comparing with lidar observations, our cloud chords are sampled from the surface, and a single cloud chord can be sampled from multiple different overlapping clouds that do not touch each other in the 3D field as hinted at by the orange chord in Fig. 3. Following Lareau et al. (2018), we define the chord-base height as the 25th percentile of the bottoms of the cloud chord grid cells. We use the term scene to refer to the $2 \mathrm{D}$ vertical slice from the surface through the cloud chord. The height of the scene is normalized from zero at the surface to 1 at the chordbase height and extends before and behind the cloud chord as shown in the two scenes shown in Fig. 3. All scenes are normalized to the same 2D grid, which allows us to merge multiple scenes together into composites. Throughout this paper we will only sort chords according to their length and do not sort them into forced or active (as done by Lamer and Kollias, 2015) or into updrafts and downdrafts (as done by Lareau et al., 2018).

In this section we detail how we define and detect cloud chords from 1D column output and 3D snapshots, followed by an analysis of how these two approaches differ. We try to be as consistent as possible with the definitions used by Lareau et al. (2018), and the exact technical implementation is described in the scripts used for this paper, which are available online through the Zenodo repository (Griewank et al., 2020). Since the model output is not identical to the measurements used by Lareau et al. (2018) we must first define some analog definitions. Lareau et al. (2018) detect cloudiness from a threshold backscatter value, whereas we consider a model grid box to be cloudy when the liquid water mixing ratio is higher than $10^{-6} \mathrm{~kg} \mathrm{~kg}^{-1}$. While we and Lareau et al. (2018) only take cloudy cells that are no more than $300 \mathrm{~m}$ higher than the convective boundary layer (CBL) into account, we must use a different definition of convective boundary layer height. This is because the vertical velocity variance used by Lareau et al. (2018) is computed from a 2D time-height slice of vertical velocity that contains many gaps in which sufficient aerosols are unavailable to retrieve a signal or liquid water attenuates the lidar too strongly. As we cannot reproduce when and where the lidar would or would not have a sufficient signal to produce a measurement, we instead use the lifting condensation level (LCL) as an additional height criterion. When the LCL is lower than the CBL height determined by the vertical velocity variance threshold, we require the cloudy cells be no more than $300 \mathrm{~m}$ higher than the LCL. Given that we do not require a high degree of precision we approximate the LCL from the mean temperature $T$ and dew point $T_{\mathrm{d}}$ of the lowest model layer following the approximation of Espy (1836), $\mathrm{LCL}=125 \cdot\left(T-T_{\mathrm{d}}\right)$, instead of using the exact expression (Romps, 2017).

In many ways it is simpler to detect chords from the simulation output than from the observations used by Lareau et al. (2018). Firstly, we know that only convective clouds occur in the LES simulations, so none of the safeguards used by Lareau et al. (2018) to minimize the chance of accidentally sampling stratus clouds are needed. Secondly, the model output contains no gaps in data to work around. Thirdly, in contrast to the observations the horizontal and vertical wind points in the model are collocated in time and space. And finally, having the full 3D domain makes it possible to accurately split the variable into a horizontal mean and local anomaly. From the observations only the local anomaly of vertical velocity can be determined easily, as we know the mean vertical velocity must be very close to zero. To calculate the observed anomalies of the water vapor mixing ratio discussed in Sect. 6 we use a 90 min running mean.

\subsection{From 1D model columns}

The 1D column outputs are created by outputting the model state in specific columns of the model grid at each time step. Each 1D column output has two dimensions, height $z$ and time $t$. Using 1D column output allows for a direct comparison with the lidar observations as both are stationary 1D measurements, which allows chords to be detected almost identically to the approach illustrated in Fig. 1 of Lareau et al. (2018). In contrast, comparing simulations to flight observations is much more difficult because it requires imitating a plane's possible flight path as discussed by Hoffmann et al. (2014).

When detecting chords from the 1D model columns we apply the same minimum chord duration of $30 \mathrm{~s}$ and maximum gap time of $20 \mathrm{~s}$ as applied to the lidar observations. But we do not apply the maximum chord duration of $20 \mathrm{~min}$, which is applied to the lidar observations. This 20 min maximum duration is one of the safeguards implemented by Lareau et al. (2018) to reduce the chance of accidentally sampling stratus clouds that are not present in the simulations.

It is noteworthy that as MicroHH does not have a constant time step, accordingly the 1D column output has a constantly varying time resolution. For reference, in one simulation the mean time step over the day is $1.2 \mathrm{~s}$ with a standard deviation of $0.2 \mathrm{~s}$. The smallest time step was $0.04 \mathrm{~s}$ and the largest $1.6 \mathrm{~s}$. On average the MicroHH time step is very close to the $1.3 \mathrm{~s}$ resolution of the Doppler lidar observations.

\subsection{From 3D model snapshots}

When calculating the chords and scenes from the 1D column output and the Doppler lidar data, we use time to turn the 1D height measurements into a $2 \mathrm{D}$ slice with height and time 


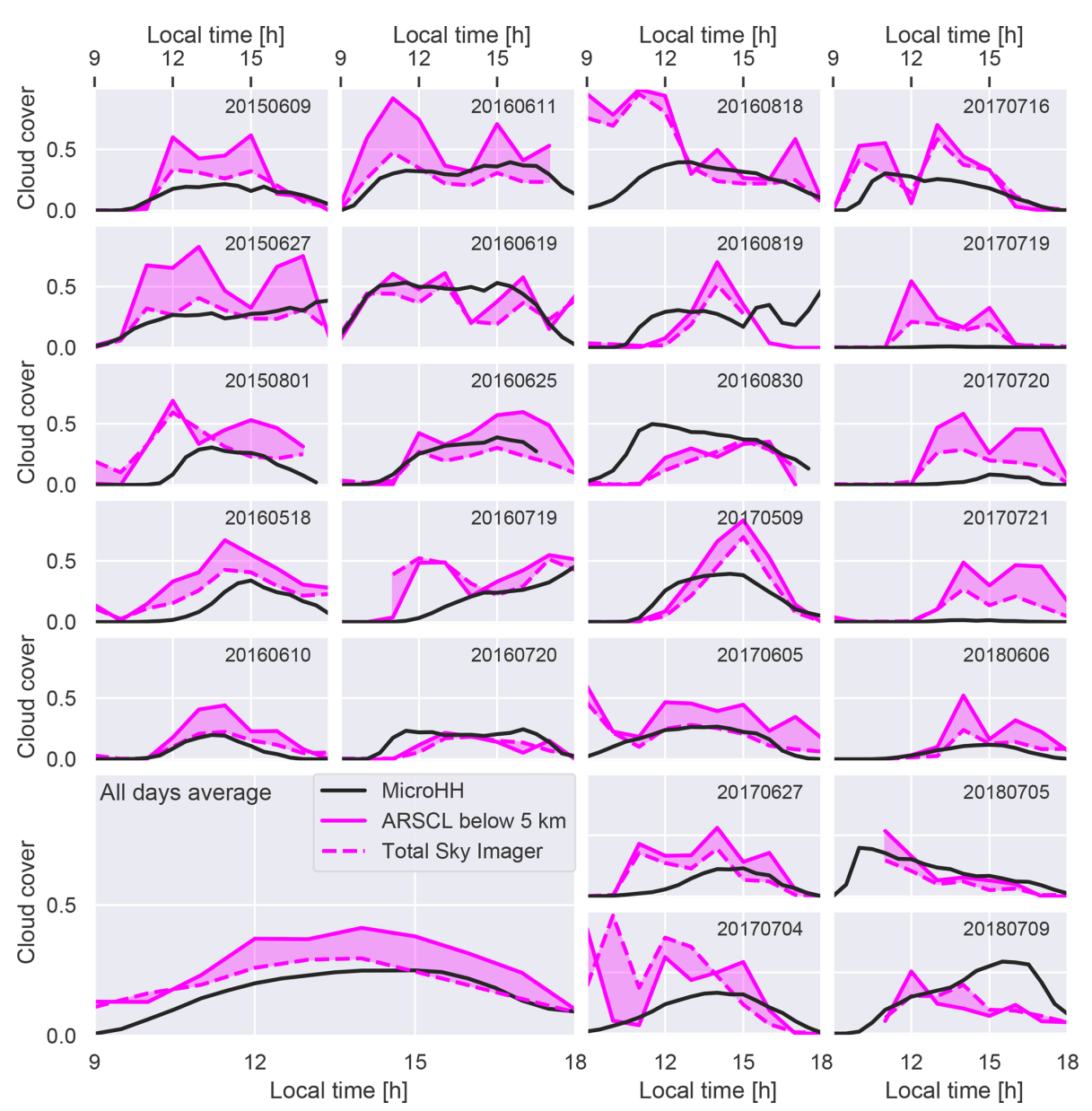

Figure 2. Daily evolution of two cloud fraction measurements provided hourly by the LASSO Bundle Browser, along with the MicroHH cloud fraction over the $25.6 \times 25.6 \mathrm{~km}$ model domain for 24 of the 28 simulations. The remaining four were not included because the cloud fraction is affected by numerical artifacts at the upper model boundary. The Total Sky Imager detects cloud fraction from a fish-eye image of the sky, while the ARSCL is a value-added product for which cloud fraction below $5 \mathrm{~km}$ is determined for 15 min windows. Observations are only used when the quality flags are ideal. The mean cloud fraction over all days is shown in the bottom left.

as the axes. In contrast, to detect chords from the 3D output snapshots we first slice the 3D field into a 2D $x-z$ slice and a 2D $y-z$ slice per snapshot. From these two slices we measure the chord length by moving an imaginary Doppler lidar across the surface. The approach of cutting in the $x$ and $y$ direction was used to detect chord length from MODIS data by Wood and Field (2011), by Endo et al. (2019) to determine $w$ across the cloud edge, and by Sakradzija and Klingebiel (2020) to determine chord length in LES output. In contrast to the chords derived from the 1D column output or the observations, we are no longer measuring the duration of the chords but the distance. We do derive a cloud duration using the mean horizontal wind speed at cloud base, but this approximation is only used as a rough comparison (shown in Fig. 6).

We also replace the $20 \mathrm{~s}$ minimum duration threshold of Lareau et al. (2018) used for the 1D and DL chords with a $75 \mathrm{~m}$ threshold, which equals three model grid cells. Since chords shorter than $250 \mathrm{~m}$ have no more than 10 cells, we do not allow gaps in the 3D cloud chords. We test in Sect. 4.3 the impact of not allowing gaps on longer chords. When calculating composite scenes we require a minimum chord length of 10 cells $(250 \mathrm{~m})$ to avoid very strong resolution differences between shorter and longer scenes.

An important characteristic of our method of slicing the 3D snapshots in the $x$ and $y$ direction is that the detected cloud chords are not aligned with the wind direction. In contrast, the 1D and Doppler lidar chords are by nature affected by the wind direction, which moves the clouds above the static sensor. Wind direction is not commonly taken into account when converting 1D measurements to cloud fractions (e.g., Brooks et al., 2005; Illingworth et al., 2007) but has a noticeable influence on our spatially connected 2D scenes. If the clouds in the domain are consistently oriented in some manner to the wind direction, this could lead to differences in chord length and duration between the 3D and $1 \mathrm{D}$ chords. 

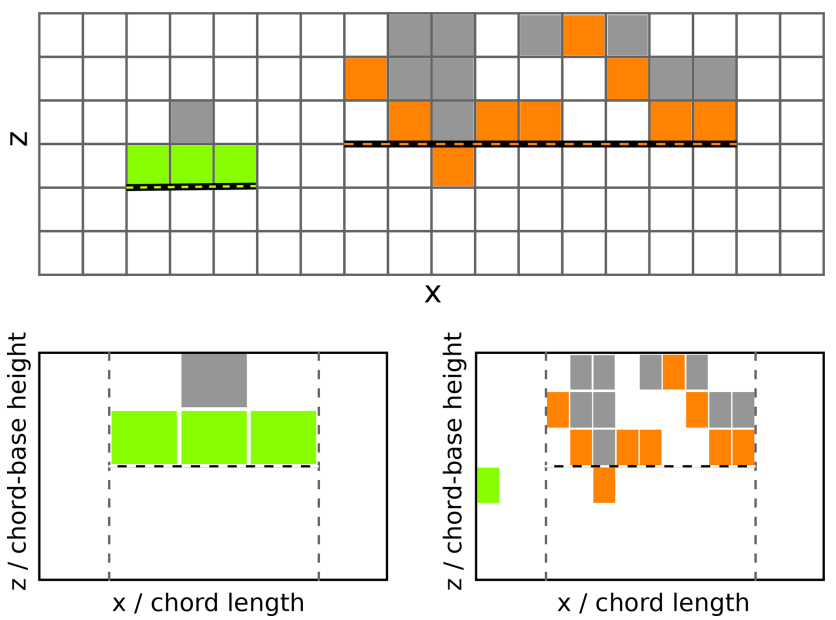

Figure 3. Illustration of chords, chord-base height, and scenes as defined in Sect. 3. The top half shows a sketch of a $z-x$ slice of 3D output. The filled-in grid boxes are cloudy cells. The green and orange cells show the cloudy cells that would be detected as two chords from the surface. The thick black lines mark the chord length and the chord-base height of the two chords. The two lower boxes show the resulting 2D scenes after normalizing the two chords with their respective chord-base height and length. For an example using Doppler lidar data, see Lareau et al. (2018, Fig. 1).

However, while we have not systematically analyzed the orientation of the clouds in regard to wind speed, we did not notice any clear formation of cloud streets or similar features in the simulations. We are confident that the various other differences in methodology between how the 1D and 3D chords are detected have a far greater effect.

While we do not take the wind direction into account when detecting chords, we try to take it into account when calculating the corresponding scenes. The method we devised to do this is by first flipping the $x-z$ and $y-z$ slices in accordance with the wind speed so that the imitation lidar moves along the surface in the opposite direction of the wind in the $x$ and $y$ direction. We then calculate and store the normalized scenes from the chords in the $x$ and $y$ direction separately for each snapshot. The last step is to weight the scenes from the $\mathrm{x}$ and $\mathrm{y}$ slices by the strength of the wind in the $x$ and $y$ direction before merging them together into one scene. The scenes from chords with differing heights and lengths can be merged thanks to the scenes being normalized in both height and distance as shown in Fig. 3.

To illustrate how the wind-weighted merging works, if the wind direction were zero in the $y$ direction, then the scene resulting from merging the weighed $x$ and $y$ scenes would be identical to the scenes in the $x$ direction. However, if the $x$ and $y$ wind components were identical, the weighted and merged scene would consist of equal parts of the scenes in the $x$ and $y$ directions. A missing but crucial detail is how we define the horizontal wind direction; for simplicity we use the mean horizontal wind direction in the 3D snapshot
Table 2. Number of chords diagnosed from model output, Doppler lidar (DL), and Raman lidar (RL) observations. There are more chords then scenes in the 3D output because we only require three cloud cells for a chord to be detected but only calculate the scene of the chord if the chord contains at least 10 cells.

\begin{tabular}{rrrrr}
\hline 3D chords & 3D scenes & 1D column & DL & RL \\
\hline 7083946 & 4249468 & 13668 & 8132 & 778 \\
\hline
\end{tabular}

at each height. An example of how taking the wind direction into account affects the resulting vertical velocity scene is shown in Fig. 4.

We acknowledge that there are other possible methods to detect chords, for example by moving the imitated lidar diagonally across the surface cutting through 2D clouds, which would result in continuous chord lengths as done by Barron et al. (2020), or we could have first rotated and interpolated the $2 \mathrm{D}$ horizontal fields onto a $2 \mathrm{D}$ grid oriented to the wind direction. However, in this paper all scenes from 3D snapshots are derived using our method introduced above because it is both technically and computationally cheaper, while avoiding any artificial interpolation artifacts.

\subsection{Differences in 1D vs. 3D}

In comparing 1D and 3D scenes and chords it is important to recognize that the DL and 1D model data are taken from slices in time, whereas the 3D snapshots that are cut into 2D slices are a function of space. Accordingly, the 1D and DL chords are sampled from clouds as they are evolving from cloud birth to death, while the 3D chords are taken from clouds frozen in time. When the horizontal wind speeds are sufficiently low, a 1D chord could contain the complete cloud life cycle from birth to death. We cannot deduce from the lidar observations alone at which stage of their life cycle the clouds are when they are sampled. Even neglecting the cloud life cycle, wind shear and rotation will stretch and deform the atmosphere as it passes over the lidar. The way we calculate scenes takes the rotation of the wind direction with height into account, but it is still a crude approximation. No postprocessing method can bridge the fundamental difference between the 1D and 3D chords. Any systematic differences between the simulated 1D and 3D chords that go beyond chord definitions should be due to the difference in sampling over space or time.

\section{Chord distributions}

This section evaluates three different aspects of the cloud chords. The first evaluation looks at chord-base height, mean horizontal velocity, and time of day. The purpose of this first evaluation is to determine how similar the distributions of atmospheric conditions are of the simulated and observed 


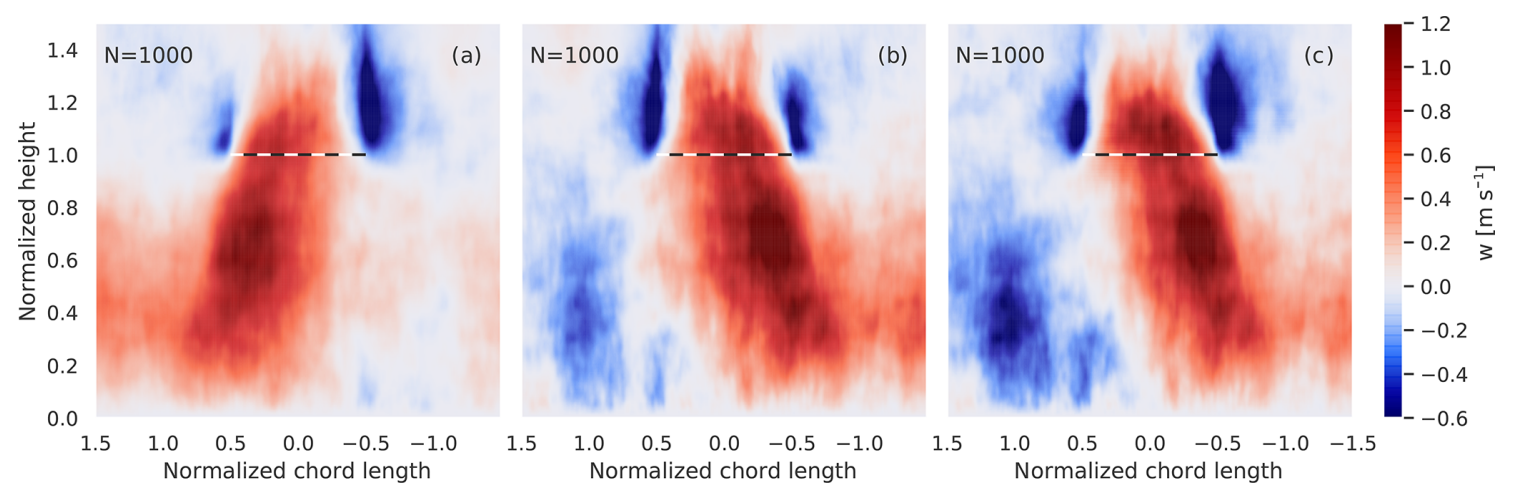

Figure 4. Mean vertical velocity scene of 500 chords in the $x$ direction and 500 chords in the $y$ direction from a 3D snapshot of the MicroHH simulation on 11 June 2016. The black dashed and white line marks the chord-base height. Shown are scenes that result from not taking the wind direction into account (a), weighting the $x$ and $y$ scenes using the wind speed at chord-base height before merging (b), and weighting the $x$ and $y$ scenes differently at each height using the vertical profile of horizontal wind (c). See Sect. 3.2 for a detailed description of how scenes are derived from 3D snapshots as illustrated in Fig. 3.

chords. The second evaluation focuses on the chord length and duration. While chord-base height and horizontal velocity are controlled by the prescribed LASSO forcing, chord length and duration are highly dependent on the resolved cloud geometry. And the cloud geometry in turn results form the simulated convective dynamics. The final evaluation is a sensitivity test to determine how much chord length and duration depend on the exact definition of cloudiness and if cloud gaps are permitted within a chord.

The differences between the 1D model chords and the Doppler lidar chords will reveal how close the geometry of the simulated cloud fields were to those measured at the ARM-SGP site, and the comparison of the $1 \mathrm{D}$ to the $3 \mathrm{D}$ model chords will reveal the differences between the temporal and spatial sampling discussed in Sect. 3.3.

\subsection{Wind, chord-base height, and time of day}

The histograms of horizontal wind speed and chord-base height both reveal significant differences between the observed and simulated chords (Fig. 5). Despite the spread being very similar the model chords are $2 \mathrm{~m} \mathrm{~s}^{-1}$ slower on average than the observed chords (mean 1D: $5.3 \mathrm{~m} \mathrm{~s}^{-1}$, mean DL: $7.2 \mathrm{~m} \mathrm{~s}^{-1}$ ). While there are an equal number of Doppler lidar chords with wind speeds below 2.5 as there are above $12.5 \mathrm{~m} \mathrm{~s}^{-1}$, the model chords rarely exceed $10 \mathrm{~m} \mathrm{~s}^{-1}$. The chord-base height distributions also do not match well. While the observed and simulated chords both have the same maximum values at around $3000 \mathrm{~m}$, there are many more model chords lower than $1500 \mathrm{~m}$. On average the model chords are $230 \mathrm{~m}$ lower.

These differences between the observed and modeled cloud chords could be attributed to two sources. Either the simulated days are not fully representative of the atmospheric conditions of the included observations, or the simulated days are not representative of the actual conditions at the ARM-SGP site at that time.

In regards to the cloud base, we have already shown that the simulations matched the observations at the ARM-SGP site very well (Fig. 1). Despite having the correct cloud-base height, the simulations could have too few clouds late in the day when clouds are higher and too many in the morning when clouds are lower. But this is not the case because the distributions of when during the day the chords were detected show no marked shift (Fig. 5c). This absence of a shift despite the average cloud cover being lower in the simulations than the observations before noon (Fig. 2) indicates that the simulated clouds are present at the right time but are not as large as their observed counterparts. Note that when screening the lidar observations for chords we exclude all clouds before 10:00 to avoid stratocumulus conditions.

In regards to the horizontal wind speed, while we cannot easily check the horizontal wind against observations because horizontal wind is not one of the LASSO evaluation observations, we find it highly unlikely that the LASSO forcing data would contain such a strong bias. Accordingly, we believe that the discrepancies between the modeled and observed chords are not due to the simulations misrepresenting the conditions at the ARM-SGP site. From this it follows that the differences are predominantly due to the $28 \mathrm{~d}$ of simulations we use not being fully representative of the wind speeds and cloud heights that result from the selection method of Lareau et al. (2018) to determine which days are shallow cumulus days.

The difference in wind speed distribution could substantially effect our analysis in three ways. First, the faster a cloud is advected over lidar the less time it has to evolve during the ongoing measurement. Second, higher horizontal speeds are typically associated with higher vertical shear of the horizontal wind, leading to greater deformation of the 
scene. Last, higher wind speeds should reduce the measured cloud duration.

\subsection{Length and duration}

In this subsection we examine the differences in chord length and duration among our three methods of chord detection. We discuss which differences can be attributed to the varying definitions used to determine a chord, the model underestimation of cloud cover noted in Sect. 2.1, or the difference in horizontal wind speed and height noted in Sect. 4.1.

We begin by comparing the duration of the Doppler lidar chords to the 1D output model chords (Fig. 6a). Both show a strong decrease in chord occurrence in regards to duration, with the most likely chord duration being just above the $30 \mathrm{~s}$ minimum time as applied by Lareau et al. (2018). The 1D chords can last longer than $20 \mathrm{~min}$, given that we did not apply any maximum time measure to the model data because there was no need to filter out stratus clouds. The distributions of the 1D chords and DL chords are remarkably similar, which at first glance would indicate that the observed and simulated clouds are of the same size and shape. However, since the horizontal wind speeds are higher than in the observations (Fig. 5), the chord length, which results from multiplying chord duration with the wind at cloud base, shows that longer chords are substantially more common in the observations than in the simulations (Fig. 6b). From this we can conclude that not only do the simulations have a lower cloud fraction as shown in Sect. 2.1,but the simulated clouds are also smaller than those observed. These findings agree with the results of Fast et al. (2019) that using homogeneous surface forcing in LES setups leads to smaller individual clouds. It is worth mentioning that the reason the most common chord length lies at roughly $300 \mathrm{~m}$ is a combination of the $30 \mathrm{~s}$ minimum duration and the mean horizontal wind speed being $5-7 \mathrm{~m} \mathrm{~s}^{-1}$. Chords shorter than $300 \mathrm{~m}$ can only be measured when the horizontal wind speed is below average.

When interpreting the 3D chords one wonders if the much larger sample size leads to a higher maximum chord length. The maximum value is expected to increase with sample size if all samples are all drawn from the same infinite distribution. However, given that the domain is $25.6 \times 25.6 \mathrm{~km}^{2}$ and the simulations rarely have a cloud fraction over 0.5 (Fig. 2), there is a limit on possible chord length (Fig. 6b). Note that the few very long chords of the order of $10 \mathrm{~km}$ are not sampled from a single $10 \mathrm{~km}$ wide cumulus cloud, but instead are the very rare occurrences when a single chord is sampled from multiple slightly overlapping cumulus clouds.

That chord occurrence decreases strongly with increasing length and duration is hardly surprising given that it has been well-established that the cumuli size density roughly follows a power law of -3 to -2 (e.g., Raga et al., 1990; Benner and Curry, 1998; Neggers et al., 2003; Zhao and Girolamo, 2007; Dawe and Austin, 2012; van Laar et al., 2019). Given that randomly cutting through a cloud with an area of $A$ cre- ates many chords with a length smaller than $\sqrt{A}$ but only a few larger than $\sqrt{A}$ (Barron et al., 2020), it is expected that the chord distribution is heavily dominated by small chords. Our results do not agree with the constant slope found by Wood and Field (2011) for chords up to $1000 \mathrm{~km}$, as Wood and Field (2011) do not exclusively look at cumulus clouds and the satellite data they used have a maximum pixel resolution of $1 \mathrm{~km}$.

\subsection{Chord definition sensitivity}

In this subsection we explore the sensitivity of the simulated chord distributions to variations in the gap tolerance and cloudy pixel definition used to define chords in the simulations. First, we examine the influence of allowing gaps in the 3D chords, which were not allowed in our baseline results. We test this by allowing $50 \mathrm{~m}$ gaps (two grid cells) and rerunning the chord detection script for $5 \mathrm{~d}$. Given that we are now allowing $50 \mathrm{~m}$ gaps we only look at chords that are at least $250 \mathrm{~m}$ long. While it is clear that allowing gaps will lead to more longer chords and fewer short chords, we cannot predict how strong that impact will be. A large impact would indicate that the simulated clouds have many small gaps in the clouds, have ragged edges, or that the clouds are often only $50 \mathrm{~m}$ away from each other. A small impact indicates that the clouds are well-isolated from each other, with few internal gaps. Our test reveals that allowing gaps has a slight but noticeable effect on chords $250-400 \mathrm{~m}$ and longer than $3000 \mathrm{~m}$ (Fig. 7a), indicating that the chords between those lengths are sampled from coherent clouds.

Second, we probe the sensitivity of the chord statistics to relaxing our cloudy pixel definition by treating cells with a relative humidity of $99 \%$ or $97 \%$ as cloudy as well. This could in theory help explain why there are more longer observed chords than detected from the 1D columns. Using a subsaturated threshold can be justified by assuming that a volume of $25 \times 25 \times 25 \mathrm{~m}$ air with such a high relative humidity could contain some condensed liquid and that by requiring liquid water to be present in the model cells to count as cloudy we might be stricter than the backscatter threshold used by Lareau et al. (2018). However, by looking at the changes in the cloud duration distribution from $18 \mathrm{~d}$ of 1D column output we see that using a relative humidity has no noticeable impact on the chord duration (Fig. 7). This strengthens our conclusion that more longer chords are observed because the simulated clouds are smaller. We did find that using a relative humidity slightly lowers cloud base but not by an amount that could impact our results.

In summary, these sensitivity tests indicate that our results are minimally sensitive to variations in the definition of cloudiness and chord continuity, indicating that the vast majority of our scenes are sampled from coherent clouds. 

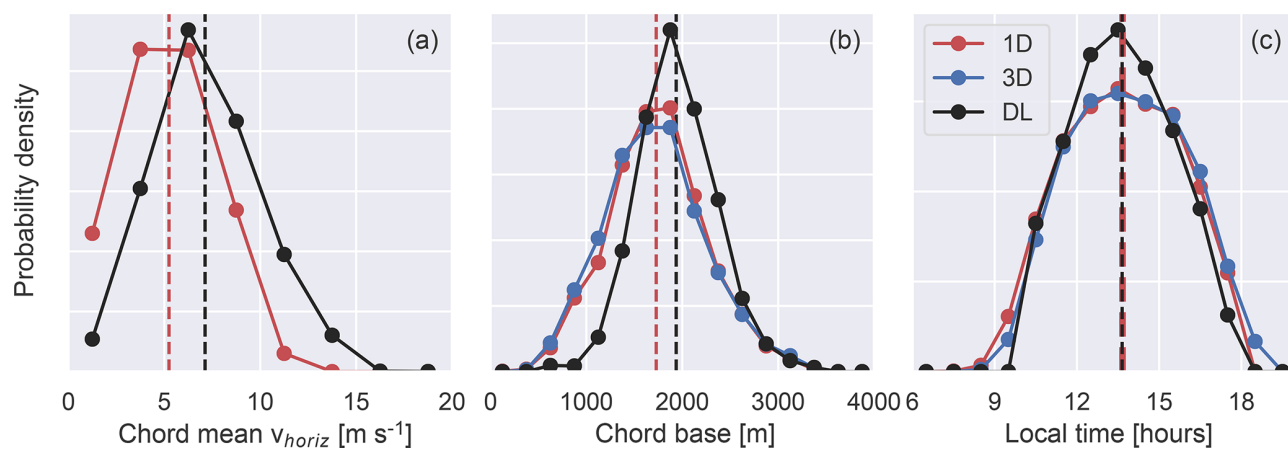

Figure 5. Probability density of the mean horizontal wind speed below the chord (a), chord-base height (b), and time of day (c) of all chords detected from the Doppler lidar, the 1D column output, and the 3D snapshots. The dashed vertical lines mark the mean values. Since the mean time of day is almost identical (13.66 vs. 13.65) the one line was plotted thicker for visibility.
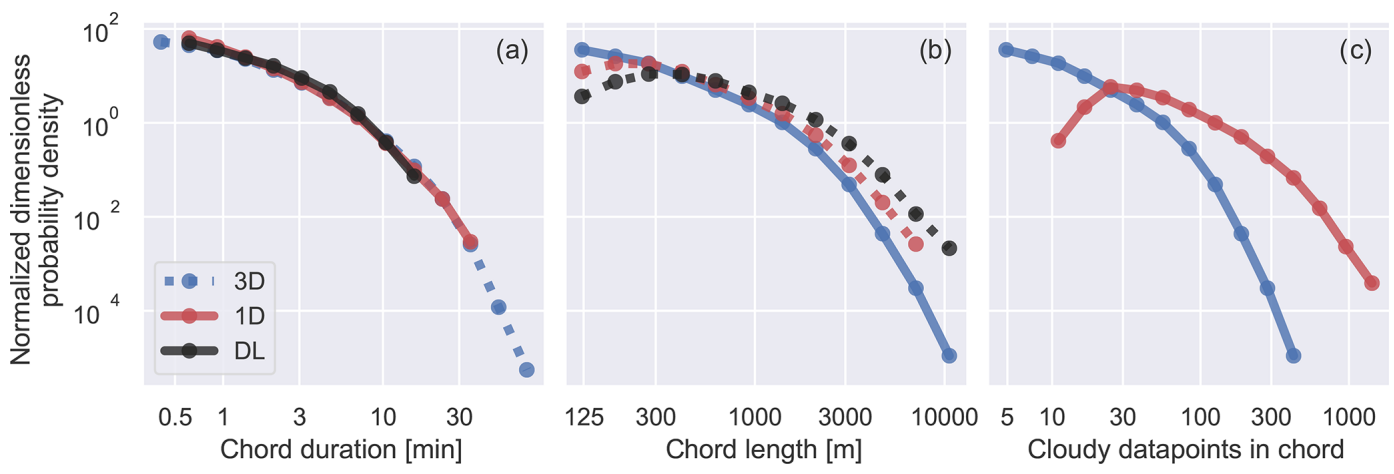

Figure 6. Distributions of (a) cloud chord duration, (b) chord length, and (c) number of cloudy data points per chord. Distributions of all chords listed in Table 2 are derived from the Doppler lidar (DL) observations, 3D model snapshots (3D), and 1D column output (1D) (description of chord detection in Sect. 3). Dashed lines indicate that the variable is derived from wind speeds.

\section{Chord vertical velocity}

Now that we have established how the distributions of the modeled and observed chords compare, we turn to the main question of our paper. Do the vertical velocities of the modeled chords have a size dependence, and does it match that of the observations? After answering that we will explore what can be learned from investigating the axial asymmetry of the $2 \mathrm{D}$ scenes.

\subsection{Size dependence}

Our analysis of the size dependence of updraft strength requires some additional data stratification. First, we restrict our analysis to chords shorter than $3000 \mathrm{~m}$ for practical and scientific reasons. Given that the cloud base at the ARM-SGP observatory rarely surpasses $3 \mathrm{~km}$ (see Fig. 1), it is likely that most chords longer than $3000 \mathrm{~m}$ are either stratus clouds that eluded the screening or a chain of smaller cumulus clouds that by chance happen to overlap. The size filtering is useful since clouds in either of these groupings do not reflect the strength to size dependence of individual cumuli.
We define the region over which to examine the mean subcloud vertical velocity $(w)$ as a box marked in each panel in Fig. 9. The box extends vertically from 0.6 to 0.8 , which we chose to capture the peak in $w$ at roughly 0.7 while staying far away enough from the cloud base to reduce the impact of the exact cloud height definition and drop contamination. The horizontal extent of the box ( -0.4 to 0.4 ) does not quite extend all the way from chord beginning to end to reduce the possibility of subsiding shells being included. We manually defined these box dimensions but checked that minor adjustments of height or width by \pm 0.05 do not effect our qualitative results.

Our representation of the subcloud velocity differs from the more commonly used $w$ at chord base, which is often considered (e.g., Endo et al., 2019) to be vertical velocity at cloud base; both are easily defined and an important property of mass flux parameterizations (Neggers et al., 2006; Sakradzija and Klingebiel, 2020). This difference is motivated by the sensitivity of chord-base height and thus $w$ at the chord base. For example, if the cloud base determined by lidar backscatter were on average $100 \mathrm{~m}$ lower than that of the cloud base detected from liquid water content in simulations, the vertical velocity at cloud base would be higher in the ob- 


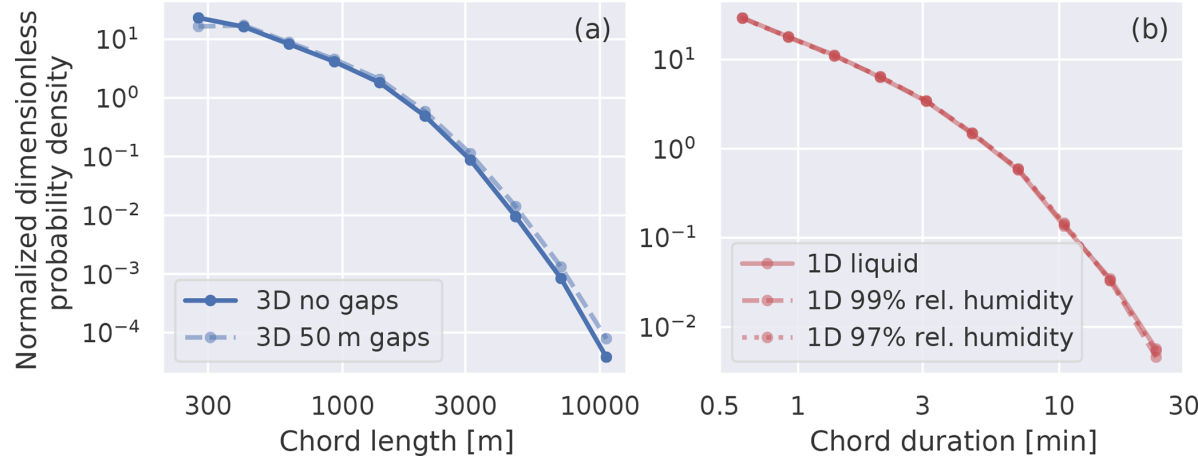

Figure 7. Distributions of (a) chord length from 5 simulations and (b) cloud chord duration from 15 simulations. The standard chord definitions introduced in Sect. 3 were modified to test the sensitivity of the results to allowing $50 \mathrm{~m}$ gaps in 3D chords (a) and replacing the cloud definition with a relative humidity threshold $(\mathbf{b})$.

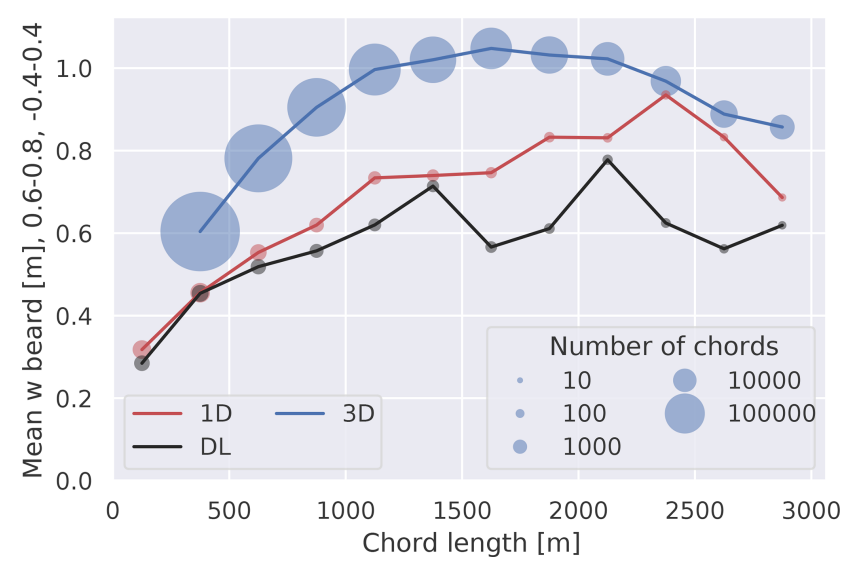

Figure 8. Mean normalized vertical velocity below chord base as a function of chord length. The area over which the vertical velocity is averaged is marked by the grey box in Fig. 9. The size of the markers represents the number of chords in that $250 \mathrm{~m}$ bin. Note that there is no $3 \mathrm{D}$ value for chords below $250 \mathrm{~m}$ as we only compute scenes for chords with at least 10 data points as explained in Sect. 2.

servations as the vertical velocity decreases with height. The second reason is due to the possibility of drops falling into the air below the cloud, contaminating the retrieved signal and leading to lower $w$. We believe this effect is at least partially responsible for the very uniform $w$ decrease visible just below cloud base (see Fig. 9d and e). We do, however, have an analysis of $w$ at cloud base in the simulations in Sect. 7.

We also opted to avoid using percentiles, such as the 95th percentile used by Lareau et al. (2018), despite the strongest updrafts being of critical importance for cloud formation. This is because the number of data points per chord is directly dependent on chord length for $3 \mathrm{D}$ chords. For example, 3D chords between 250 and $500 \mathrm{~m}$ only have 10-20 data points (Fig. 6c). This increase in sampling size is also true for the 1D and DL chords to a lesser extent. Due to the large change in sampling size, calculating the mean over the 95th percentiles of chords with different lengths leads to an artificial signal.

The first result of our analysis is that the $1 \mathrm{D}$ and DL chords agree well for chords shorter than $1500 \mathrm{~m}$ (Fig. 8), although a consistent bias exists within this range. The simulated chords have stronger updrafts at all lengths, with the difference between model and observation increasing to $\approx 0.2 \mathrm{~m} \mathrm{~s}^{-1}$ for chords between 1500 and $3000 \mathrm{~m}$ for which sampling is the weakest. The bias is less than that found by Endo et al. (2019) at cloud base, which we attribute to the differences in our definition of the subcloud velocity (i.e., at cloud base vs. in the subcloud box). We also note that the $w$ probability density function (PDF) at cloud base studied by Endo et al. (2019) is not sampled from cloud chords but from all cloud-base grid cells, so the distinction between 1D and 3D sampling has no effect on their findings. The general shape of the 1D curve in Fig. 8 is consistent with the findings of Ansmann et al. (2010) and Lamer and Kollias (2015). A quantitative comparison is not possible since Ansmann et al. (2010) studied updrafts at a fixed height within the boundary layer, and Lamer and Kollias (2015) compare the updrafts and downdrafts of the cloud chords separately, as well as normalized chord length by cloud-base height and vertical velocity by the convective velocity scale $\left(w^{*}\right)$.

While it could be argued that the 1D and DL chords have the same behavior for chords shorter than $1500 \mathrm{~m}$, the differences between the 1D and 3D chords are much more pronounced (Fig. 8). The 3D chords show a stronger scaling until $1000 \mathrm{~m}$, with a weak signal beyond but with a distinct small peak at $1500-1750 \mathrm{~m}$.

In conclusion, 1D and DL chords are in good agreement for chords shorter than $1500 \mathrm{~m}$. While a modest bias exists between $1 \mathrm{D}$ and DL in that range, it is significantly smaller than the increase in the mean $w$ across that range. In addition, this bias is much smaller than the difference between the 1D columns on the one hand and 3D snapshots on the other. All three chord measures show a marked increase in $w$ in the first kilometer; while the 1D model chords show a clear size de- 
pendence up to $2000 \mathrm{~m}$, the 3D chords level off after $1000 \mathrm{~m}$, and the signal in the observations is only beyond a doubt for the first $1000 \mathrm{~m}$.

\section{$5.2 w$ scene}

In this subsection we examine the size and shape sensitivity of the broader subcloud vertical velocity scene shown in Fig. 9. The analysis uses the time-height normalized com-

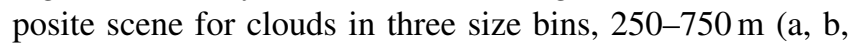
c), 750-1500 m (d, e, f), and 1500-2500 m (g, h, i).

Figure $9 \mathrm{a}, \mathrm{d}$, and $\mathrm{g}$ are similar to those included in the supplementary material of Lareau et al. (2018) but contain more data and are binned differently. Note that the observed data are limited to regions where $>70 \%$ of the observations have sufficient aerosol and are cloud-free, reducing the available data above 0.8 of cloud-base height on the scene periphery. Since we do not know if the occurrence of sufficient aerosol at cloud-base height is independent of $w$, we are not fully confident in the observed vertical velocities above 0.8 on the scene periphery.

In addition, we are wary of DL data close to cloud base ( $>0.9$ beneath the chord) due possible interactions with cloud droplets. For example, we suspect that the reduction to 0 in vertical velocity at cloud base in Fig. 9a, d, and $g$ is an artifact of the cloud droplets impacting the $w$ retrieval. This is supported by the simulated scenes not having such a feature at all and also there being no theoretical reason for updrafts to stop at cloud base.

Figure 9 indicates that the simulations and observations disagree in two aspects. Firstly, the 1D scenes have no downdrafts extending below 0.8 normalized height (Fig. 9b, e, h). This could indicate that the model resolution, microphysics, and/or 1D radiation are insufficient to fully capture the evaporative cooling at the cloud edge (Abma et al., 2013), which agrees with the findings of Endo et al. (2019). Secondly, the vertical velocity is higher in the simulated chords longer than $750 \mathrm{~m}$ as discussed in Sect. 5.1. But otherwise the 1D and DL scenes show a very similar pattern and extent. Both have stronger downdrafts in the wake of the chord, with the strongest updraft located at roughly 0.7 normalized height with a marked shift to the right; i.e., the chords have a stronger updraft earlier on. Not visible in the observations is that, as expected, the updrafts of the longer chords extend to a greater altitude.

While we cannot fully isolate what causes the differences between the 1D and DL scenes, we find that the following general picture has emerged from our analysis. On average the simulated clouds are more compact and closely linked to convective plumes than the observed plumes. Given how small the differences are for shorter chords there are many possible explanations, such as the difference in horizontal wind shown in Fig. 5 or the observations including some days with anomalously weak convection. In addition, the longer observed chords appear to have a weaker convective charac- ter (Fig. 9g) and show no clear link between chord length and updraft strength. This could indicate that some of the longest observed cloud chords are stratus clouds that eluded our selection criteria intended to filter stratus clouds. But we cannot check if this occurs at all, let alone sufficiently often to have a noticeable effect on the mean value.

In contrast to the DL and 1D scenes, the 3D chords are more symmetric with only a weak shift of the updraft core towards the start of the chord (i.e., in Fig. 9c, f, i). The symmetry is more pronounced when looking at the two subsiding shells at the edge of cloud chords, which are equally strong and extend to the same height. There is a slight asymmetry in that the downdraft region behind the chord is a bit wider, but not to the extent Mallaun et al. (2018) detected in airplane measurements. Also, in contrast to the 1D scenes the updraft is more tightly organized below the cloud chord. This is most clearly visible when looking at how far the updrafts trail behind the chord, i.e., in Fig. 9a, d, and g.

Returning to the differences between 1D and 3D chords introduced in Sect. 3.3, the difference between 1D and 3D can be attributed to either the life cycle of the cloud evolving as it is sampled or the upwind advection stretching the scene as it is advected over the lidar. The shear in horizontal wind speed over the boundary layer can explain why the updrafts in the 1D and DL chords are broader below the chord (Heus and Jonker, 2008). Weaker horizontal winds closer to the surface would laterally stretch the updrafts, causing the wide weak updrafts close to the surface in the 1D and DL scenes. A wind sheer above the chord base should be visible by the scene above the chord being slightly pulled toward the center of the chord. A close look at the trailing subsiding shell in the $1 \mathrm{D}$ chords shows that the subsiding shell bends slightly to the right over the cloud chord (Fig. 9b, e, h). In contrast, the subsiding shells of the 3D chords extend straight upwards. While this is only a very weak signal, it is consistent with what we would expect from a slight horizontal wind increase above the cloud base.

The asymmetry, however, cannot be explained by the vertical shear of the horizontal wind. Instead, our results suggest that the asymmetry arises from the ongoing cloud life cycle. Given that the average horizontal wind speed is about $6 \mathrm{~m} \mathrm{~s}^{-1}$ (see Fig. 5), on average we would expect the smallest clouds to age $1.5 \mathrm{~min}$ from chord beginning to end, the middle chords $3 \mathrm{~min}$, and the longest chords $5.5 \mathrm{~min}$ (Fig. 9). Given that most shallow cumulus clouds live less than $20 \mathrm{~min}$ and many far shorter (Dawe and Austin, 2012; Heus and Seifert, 2013), the clouds are substantially younger at the beginning of the chords (i.e., Fig. 9c, f, i) than at the end. We expect younger cumulus clouds to be growing with an active updraft and older clouds to be decaying and sinking. Knowing that the chords are substantially older at the end of the chord, this aging provides a consistent explanation for why updrafts both below and above the chord base are stronger at the beginning of the cloud chords. 


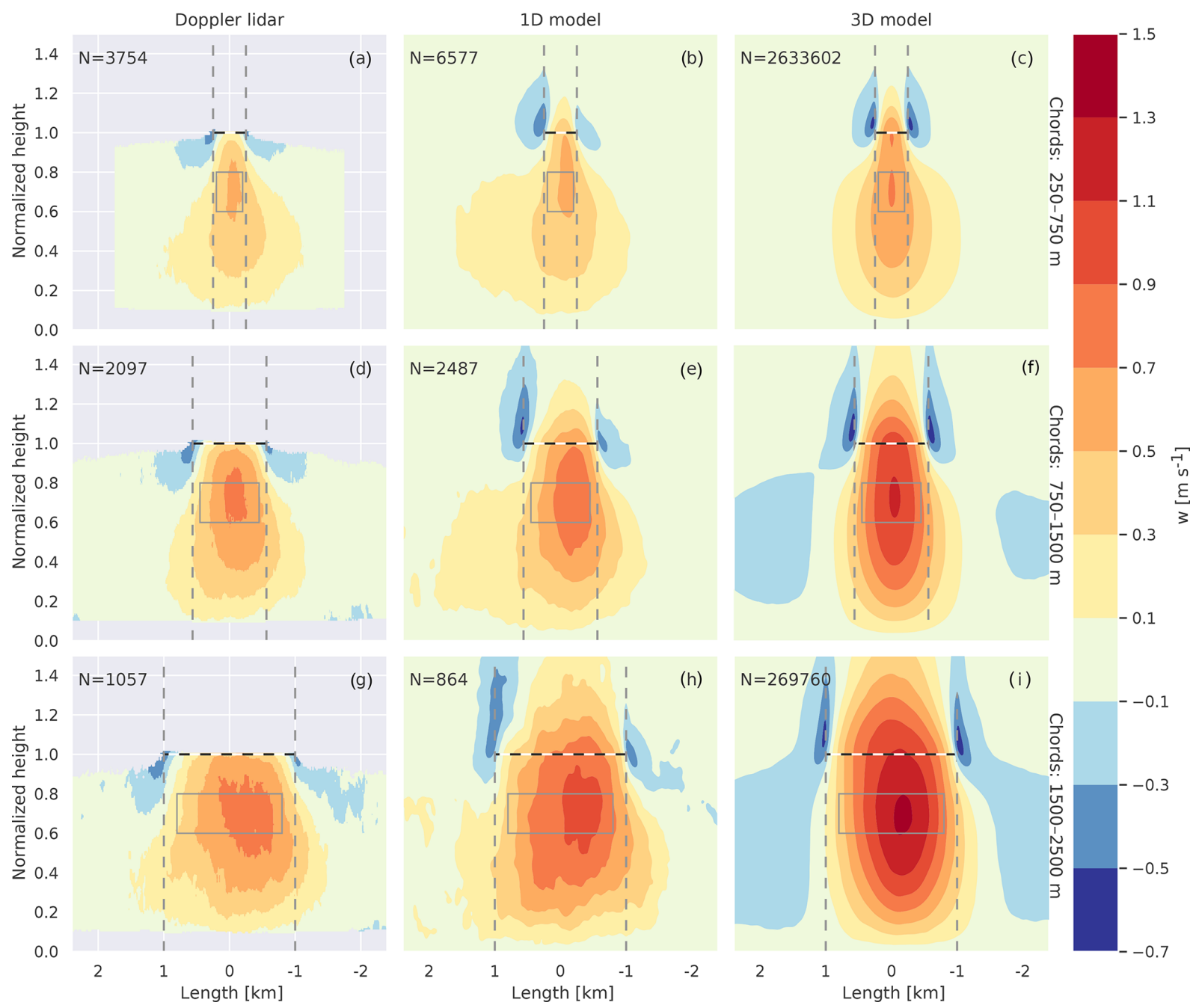

Figure 9. Normalized vertical velocity scenes from Doppler lidar observations (a, d, g), 1D column output (b, e, h), and 3D snapshots (c, f, i). The scenes are composites of the normalized scenes binned by chord lengths of 250-750 m (a, b, c), 750-1500 m (d, e, f), and 1500$2500 \mathrm{~m}(\mathbf{g}, \mathbf{h}, \mathbf{i})$. After merging the scenes the normalized length is rescaled to match the mean length of the bin (e.g., $500 \mathrm{~m} \mathrm{for} 250-750 \mathrm{~m}$ ). The black dashed and white horizontal line marks the chord base, the dashed grey lines the chord beginning and end, and the grey box the area averaged for Fig. 8. See Sect. 2.1 for information on the Doppler data and Sect. 3.2-3.1 for descriptions of how chords were derived from the MicroHH simulations. Doppler lidar scenes are only shown when at least $70 \%$ of the scenes contain data.

Having considered possible explanations for the differences in width and asymmetry between the $1 \mathrm{D}$ and DL chords, we have so far not explained why the 3D chords have stronger updrafts. The difference in updraft strength is very likely partially due to the 3D chords not allowing any gaps in the chords. While we have shown that allowing $50 \mathrm{~m}$ gaps does not effect our results (Sect. 4.3), we know that allowing longer gaps over hundreds of meters does reduce the updraft velocity. Interestingly, not only are the 3D updrafts stronger, but the downdrafts behind and before the chords are also stronger for the biggest chords (Fig. 9i). Given that the long chords have a strong updraft, due to mass conservation in the LES domain there must be downdrafts between the chords. That these downdrafts are not visible in the 1D chords indi- cates that while the 3D chords taken from the frozen snapshots have a clear separation of updrafts beneath the clouds and subsidence between, this clear separation is blurred by the ongoing time evolution in the $1 \mathrm{D}$ chords.

\section{Moisture anomalies}

After comparing the simulated vertical velocities against the Doppler lidar measurements, we now move on to the moisture anomalies computed from the Raman lidar. Our core question remains, which is to what degree the model and observations agree and if the anomalies show a clear size dependence. 
For interpreting the Raman lidar results it is important to note that the sample size and frequency of the moisture anomalies are substantially lower than those of the vertical velocity. We have roughly a factor of 10 fewer observed chords (Table 2) and the sample frequency is also lower by a factor of 10 (10 s vs. $1.3 \mathrm{~s}$ ). To make use of all the data we have available for the largest chords we expanded the binning of the scenes shown in Fig. 10 compared to Fig. 9 (1500-3000 m vs. 1500-2500 m). While the Raman lidar observations tend to extend higher than the CBL, they cannot penetrate into the clouds (Lareau, 2020). Since we calculate the anomalies from a 90 min running mean we do not plot the anomalies higher than 0.95 of the chord base because the missing observations in the cloud bias the resulting anomalies.

As expected, due to the sharp decrease in water vapor in the atmosphere above the boundary layer, both observed and simulated moisture anomalies have a very strong maximum at cloud chord base and, for the simulations, in the cloud cores (Fig. 10). In both the observations and simulations the positive moisture anomaly reaches all the way to surface, with a smooth and monotonic increase from the surface to cloud base. In our simulations the water vapor values in the lower mixed layer lie roughly between 10 and $15 \mathrm{~g} \mathrm{~kg}^{-1}$, so the mean anomalies at 0.5 chord-base height are on the order of $1 \%-2 \%$. To avoid the signal being dominated by possible small shifts in cloud height, we use a larger and lower averaging box (marked in grey in Fig. 10) to calculate the moisture anomalies shown in Fig. 11 than we did for the vertical velocities (marked in grey in Fig. 9).

In contrast to the vertical velocity, which turns negative within the cloud edge (Fig. 9), the water vapor anomalies remain slightly positive beyond the cloud at the cloud-base height. This is consistent with our general understanding of subsiding shells, which, while drier than the cloud cores, are still moister than the surrounding air (Heus and Jonker, 2008; Wang and Geerts, 2010; Katzwinkel et al., 2014; Lareau, 2020).

Comparing the RL and 1D moisture anomaly scenes to the 3D scenes results in the same conclusions we reached from the vertical velocity scenes. The RL and $1 \mathrm{D}$ chords have a clear asymmetry with no clear negative anomalies before and behind the chords, while the 3D chords are almost symmetric with clear dry areas before and behind the chords (Fig. 10).

The size dependence of the moisture anomalies below the chord base of the 1D and 3D scenes is also quite similar to that of the vertical velocity (Fig. 11). Again the 3D scenes have stronger anomalies than the 1D scenes, with the highest anomalies corresponding to chords that roughly have the length of the mean boundary layer height $(1.5 \mathrm{~km})$, while the 1D scene anomalies slowly increase with chord length until about $2.5 \mathrm{~km}$. In contrast to the simulated scenes the observed moisture anomalies decrease with length, although there are large fluctuations between bins due to the small sample sizes. It should be noted that the relative anomalies are only in the range of $1 \%-2 \%$ and that any bias or error in the anomaly calculation could substantially effect the results. To more completely understand the size dependence in the observations would likely require substantially more data, preferably with a higher measurement frequency, and a more careful separation of anomalies from the mean than what we use. However, for now we are confident that the decreasing moisture anomalies with length are a direct result of using a running mean to calculate the anomalies.

In summary, the moisture anomaly analysis shows that the simulations match our theoretical expectations while correctly reproducing the observations. The differences between the 1D and 3D scenes are consistent with our findings from the vertical velocity in that the $1 \mathrm{D}$ chords have a weaker signal with asymmetric scenes. Both $1 \mathrm{D}$ and 3D scenes show a clear size dependence, while the observed chords have a weak inverse size dependence due to how the anomalies were calculated. In relative terms the anomalies halfway below the chords are in the range of $1 \%-2 \%$.

\section{Sampling uncertainty}

In this section we use simulated data to address the question of how the uncertainty in cumulus properties depends on how many days of shallow cumulus lidar observations are available. Assuming a single 1D column output of a shallow cumulus simulation is equivalent to having one lidar measuring over $24 \mathrm{~h}$; this analysis will determine how many days of shallow cumulus lidar observations would be needed at the ARM-SGP site to derive the various chord properties to within a threshold uncertainty. This analysis is based on the premise that each individual column output is both independent and equivalent; i.e., we make no distinction between two column outputs from the same day or different days. On average 31 chords are detected from each column, with a standard deviation of 17 and a maximum of 68 . For this analysis we exclude all columns with fewer than 10 chords, leaving us with 386 columns of our total 448 . The three properties that will be investigated are the chord-length distribution, the mean vertical velocity below the chords as a function of chord length, and the mean $w$ scene.

The sampling uncertainty of the chord-length distribution for $n$ columns is determined by repeatedly sampling $n$ columns at random and calculating the chord distribution of all the chords contained in those $n$ columns. As expected, uncertainty in the chord-length distribution is directly linked to chord occurrence, with the smallest uncertainty being at about $300 \mathrm{~m}$, which is the most common chord length (Fig. 12a). At this point it is informative to compare these results with the DL observations. The line presenting the DL chords roughly lies within the $n=64$ shading, which implies that with fewer than 64 columns we cannot rule out the possibility that the differences between the observed and simulated chord-length distributions are due to sampling. 

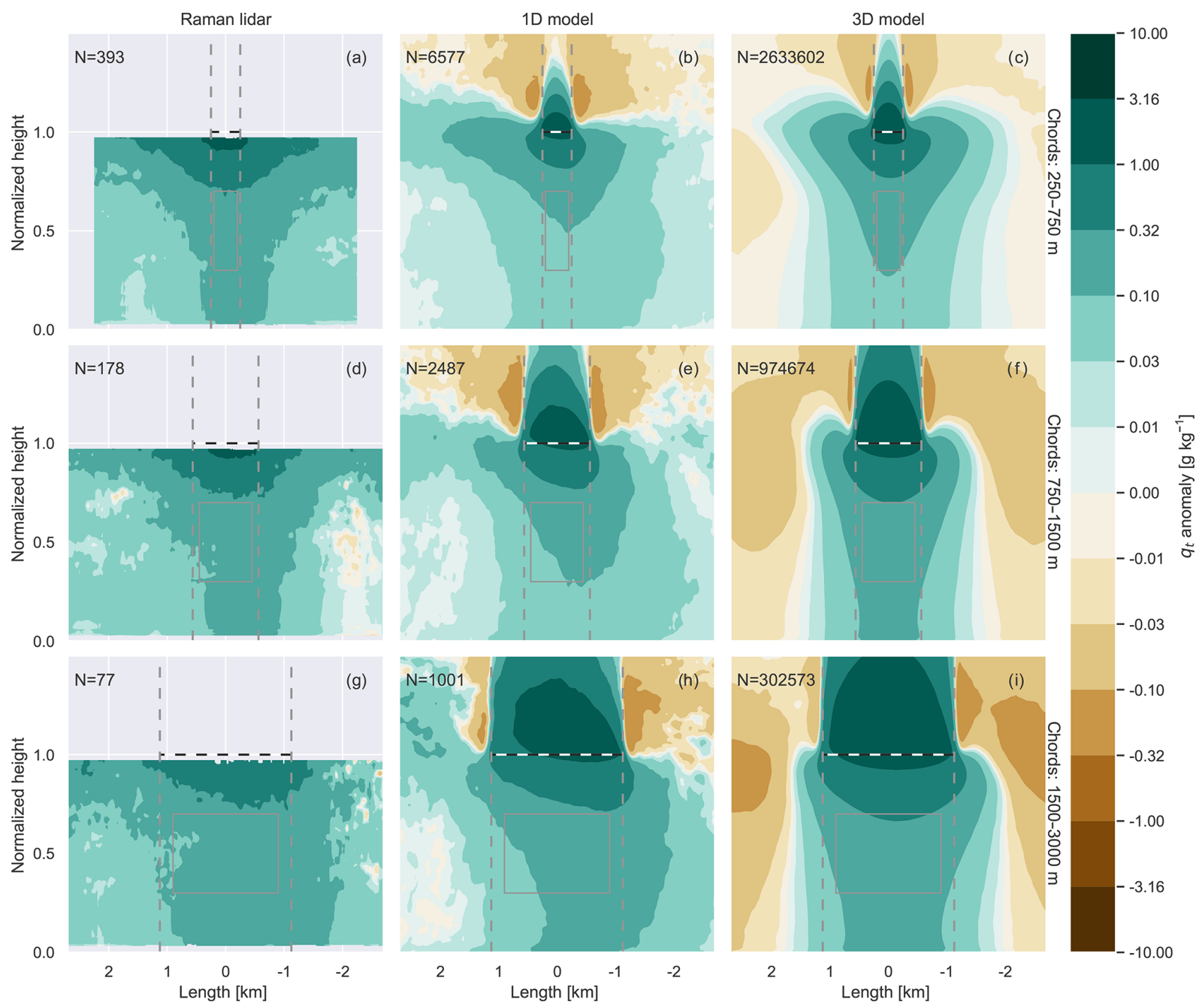

Figure 10. Scenes of $q_{t}$ anomalies $\left(\mathrm{g} \mathrm{kg}^{-1}\right)$ from Raman lidar observations, 1D column output, and 3D column output. The scenes are composites of the normalized scenes binned by chord lengths of 250-750 m (a, b, c), 750-1500 m (d, e, f), and 1500-2500 m (g, h, i). After merging the scenes the normalized length is rescaled to match the mean length of the bin (e.g., $500 \mathrm{~m}$ for $250-750 \mathrm{~m}$ ). The black dashed and white horizontal line marks the chord base, and the dashed grey lines mark the chord beginning and end. See Sect. 2 for information on how the scenes are computed. Note the logarithmic color bar. For reference, the water vapor values in the lower mixed layer range from 10 to $15 \mathrm{~g} \mathrm{~kg}^{-1}$.

The mean $w$ at cloud base is displayed in the same manner as the chord-length distribution (Fig. 12b). In contrast to chord length, the uncertainty regarding the dependence of mean $w$ at chord base on chord length is relatively constant for chords from 100 to $3000 \mathrm{~m}$. We speculate that two compensating effects are behind this behavior; while longer chords are rarer than shorter chords, their mean $w$ also has a much smaller spread. Accordingly, fewer long chords are needed to reach a representative mean value. An interesting result not directly related to sample sizes is that the shortest chords below $200 \mathrm{~m}$ in length actually have a negative vertical velocity on average. This negative velocity for the smallest chords was also found by Rodts et al. (2003) in airplane measurements and indicates that the smallest chords are dominated by chords sampled from the subsiding shells of larger clouds or from dying clouds at the end of their life cycle.

The final variable we examine is the $2 \mathrm{D} w$ scene. To determine the uncertainty in the $w$ scene we calculate the root mean square error (RMSE) between two randomly sampled scene composites through a bootstrapping approach. The randomly sampled scenes are generated by drawing $2 \cdot n$ of columns into two separate groups with $n$ columns each from our total pool of 386 1D columns (again we only include columns that contain at least 10 chords). Once a column is drawn it is removed from the pool so that no column is compared against itself. We then form a composite of all $w$ scenes present in the $n$ columns in each group. Finally, we calcu- 


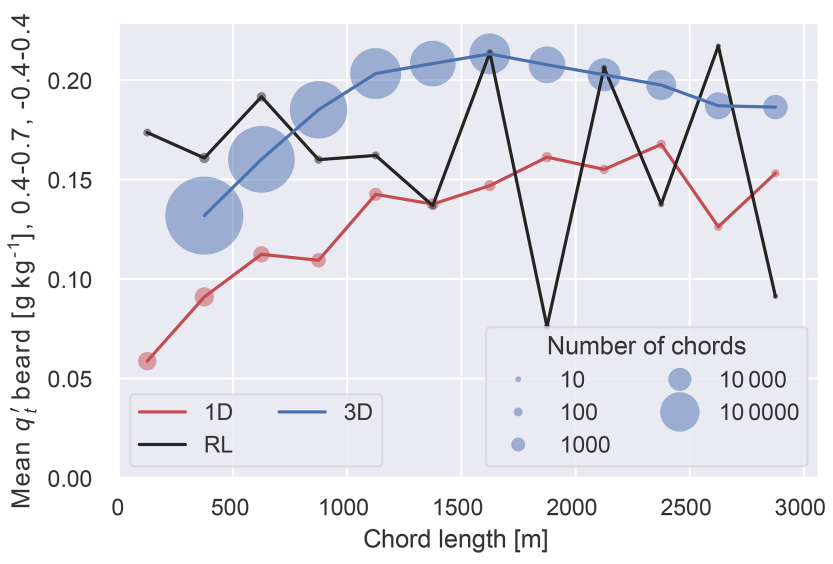

Figure 11. Mean water vapor anomalies below chord base as a function of chord length. The area over which the anomaly is averaged is marked by the grey box in Fig. 10. The size of the markers represents the number of chords in that $250 \mathrm{~m}$ bin. Note that there is no 3D value for chords below $250 \mathrm{~m}$ as we only compute scenes for chords with at least 10 data points as explained in Sect. 2.

late the RMSE between the two $w$ scenes that were calculated from the two pots. This random drawing is repeated 100 times. Note that the selection of 100 permutations is arbitrary, but the results remain unchanged for higher numbers.

As each column contains a varying number of scenes with different lengths and from varying times of day, there is no easy way to predict how the RMSE will behave. Our analysis shows that on average the RMSE behaves as if drawing from normally distributed errors with a standard deviation of $0.3 \mathrm{~m} \mathrm{~s}^{-1}$ (Fig. 13). Accordingly, quadrupling the columns included in the analysis halves the sampling uncertainty. For example, to reduce the expected sampling uncertainty from 0.05 to $0.025 \mathrm{~m} \mathrm{~s}^{-1}$ requires measuring $128 \mathrm{~d}$ instead of 32 .

\section{Conclusions and discussion}

The two main goals of our study were to evaluate the LES concerning (thermo)dynamic perturbations of continental transient shallow cumuli and to establish if size dependence exists in these features. These goals were achieved by comparing cloud chords observed by Doppler and Raman lidars against chords derived from $1 \mathrm{D}$ and 3D model output. From our results we established the following.

\subsection{Conclusions}

- We are the first to show that an LES approach can reproduce the magnitude and shape of vertical velocities and moisture anomalies observed below shallow cumulus clouds.

- The size dependence of vertical velocity on chord length is clear and robust. Our work puts the findings of Rodts et al. (2003), Lamer and Kollias (2015), Neggers

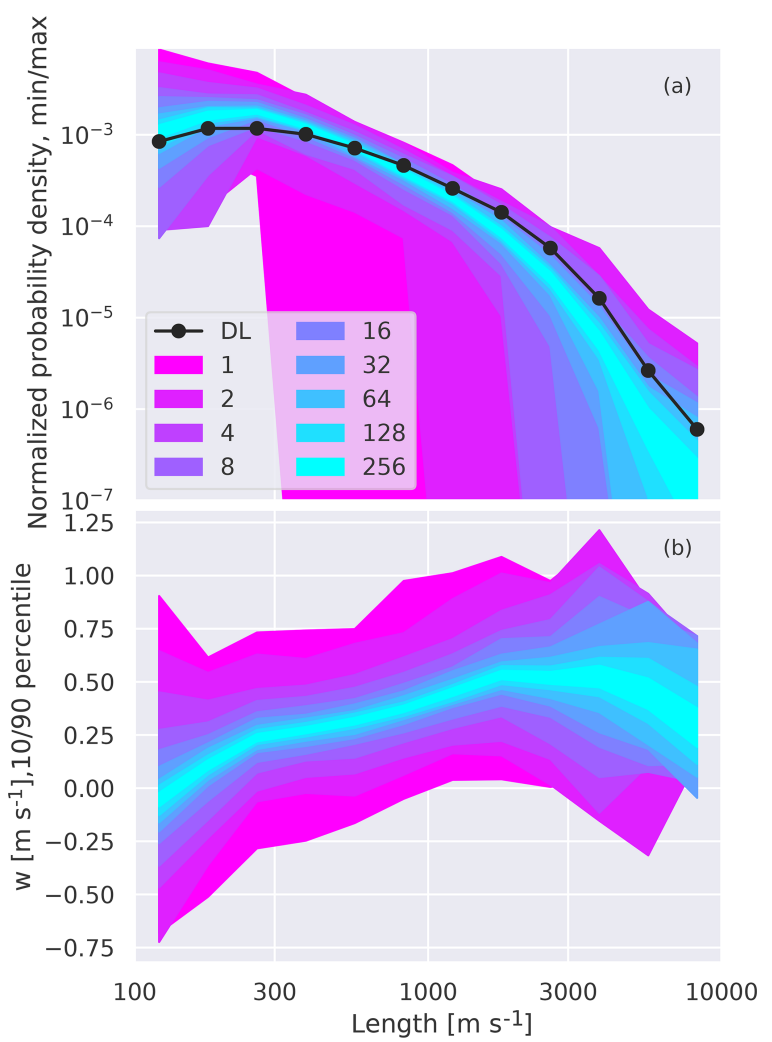

Figure 12. Chord-length distribution (a) and mean vertical velocity below the chord binned by chord length (b) as a function of chord length based on simulation data. The shading in (a) shows the minimum or maximum value that results from randomly sampling and combining $n$ columns 386 times (details in Sect. 7). The shading and legend mark how many $n$ 1D column outputs were combined together in each random sample. The shading in (b) fills the 10th to 90th percentile of all the samples. The black line is the Doppler lidar data, which shows roughly the same number of chords as we detect from 250 column outputs.

(2015), and Lareau et al. (2018) on a more robust statistical foundation.

- Compared to observations, MicroHH in combination with the LASSO forcing leads to a lower cloud fraction (similar to Schalkwijk et al., 2015; Zhang et al., 2017; Gustafson et al., 2020) and slightly shorter cloud chords.

- We see a positive bias in modeled vertical velocity at roughly 0.7 chord height that is smaller than $0.1 \mathrm{~m} \mathrm{~s}^{-1}$ for chords shorter than $1500 \mathrm{~m}$. Our bias is an order of magnitude smaller than the bias noted by Endo et al. (2019) at cloud base.

- Differences between the observations and simulations are smaller than the differences between the 1D and 3D sampling approaches applied to the simulations. 


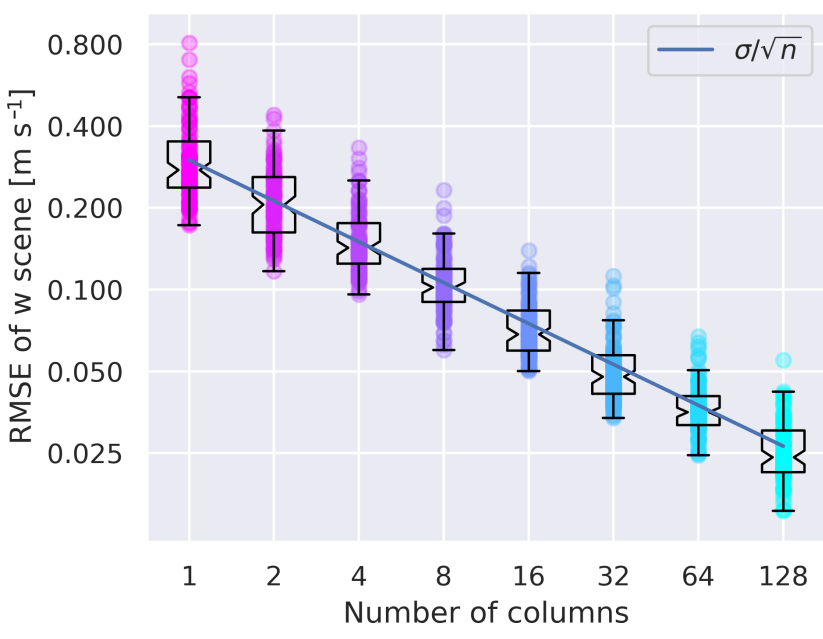

Figure 13. Root mean square error (RMSE) between two randomly sampled $w$ scene composites. The exact process by which the sampling occurs is explained in Sect. 7 and is applied 100 times for each color. The $x$ axis denotes the number of 1D column outputs included in the randomly sampled scenes. Note the logarithmic $y$ axis. The added line marks the sampling error associated with drawing $n$ samples from a normal distribution with a standard deviation of $0.3 \mathrm{~m} \mathrm{~s}^{-1}$.

- The quantitative analysis of the observed moisture anomaly dependence on chord size is complicated by the relatively weak anomalies, the need to determine the mean moisture, and the smaller data amounts available from the Raman lidar. For now, solving this problem is considered a future research effort.

- Our results suggest that the asymmetry in the observed Doppler lidar scenes mainly originates from the cloud evolving while it is being sampled.

- Roughly $60 \mathrm{~d}$ of Doppler lidar observations from the ARM-SGP site are needed to detect the differences between observed and simulated chord-length distribution with certainty.

\subsection{Discussion}

Our success at using measurements to evaluate LES performance in combination with using LES output to better understand the measurements at the ARM-SGP site underlines the usefulness of initiatives to run continuous LES experiments at super-sites such as LASSO (Gustafson et al., 2020) and the JOYCE test bed (Neggers et al., 2012; van Laar et al., 2019) to drive scientific progress. Our methodology also highlights again just how crucial it is to imitate the observations as closely as possible when evaluating LES experiments against observations and illustrates one of the many meaningful ways LES output can be analyzed beyond looking at profiles of fluxes and mean variables. The underestimation of cloud fraction and cloud size in the simulations matches the recent findings of Fast et al. (2019) that using a horizontally homogeneous surface moisture reduces cloud size and lifetime.

Our composites of millions of scenes taken from the 3D snapshots indicate that on average the shape of a standard symmetric and cylindrical convective plume as originally formulated by Simpson and Wiggert (1969) is a reasonable approximation, despite individual clouds being asymmetric (Zhao and Austin, 2005) or composed of a collection of thermal bubbles (French et al., 1999; Yano, 2014). The clear scaling in vertical velocity below the clouds supports the use of multiple plumes with different sizes as originally proposed by Arakawa and Schubert (1974). Also in regards to parameterized plumes, that the moisture anomalies below the clouds are relatively small (1\%-2\%) indicates that initializing multiple plumes at the surface using the background moisture is justifiable (Park, 2014; Neggers, 2015; Hagos et al., 2018).

While we expect our results from the ARM-SGP site to qualitatively apply to continental shallow cumulus elsewhere, the applicability to cumulus convection in different climate regimes (e.g., marine trade wind, mixed phase, high latitude) and in different modes (precipitating, deep) still needs to be demonstrated. Now that we have shown that the LES outputs capture the observed vertical velocity and moisture structure of shallow cumulus chords at the ARM-SGP site, we can confidently use the LES experiments to study features linked to shallow cumulus dynamics, such as convective plumes, subsiding shells, and cold pools.

Code and data availability. The simulation and lidar data shown in the figures are freely available at https://doi.org/10.5281/zenodo.3731944 (Griewank et al., 2020), which also contains the Python files to plot the data and post-process the simulations. The simulations were generated with version 1.9 .1 of $\mathrm{MicroHH}$ (https://github.com/microhh/microhh2/releases/tag/1.9.1, Heus, 2020). The data used to force MicroHH and to evaluate the simulated cloud fraction and base are available through the LASSO Bundle Browser (https://adc.arm.gov/lassobrowser, ARM, 2020a). The lidar data that were processed are freely available through the ARM data archive at https://adc.arm.gov/data/ (ARM, 2020b) (see Sect. 2.1).

Author contributions. All authors contributed substantially to the research direction and scope, paper structure, and conclusions over a 2-year timeframe. PJG wrote the vast majority of the text, postprocessed the simulations, and plotted all the figures. TH set up and ran the simulations. NPL gathered and post-processed the observations and helped proofread. RAJN provided experience and context to interpret the results in regards to cumulus parameterizations and LES evaluation at meteorological super-sites. 
Competing interests. The authors declare that they have no conflict of interest.

Acknowledgements. Our research would not have been possible without the groundwork laid by the LASSO project (LES ARM Symbiotic Simulation and Observation) and the Southern Great Plains (ARM-SGP) atmospheric observatory established by the Atmospheric Radiation Measurement (ARM) user facility. We would like to thank the University of Cologne for funding a mobility grant used by Philipp Griewank to visit Thijs Heus and Neil Lareau. The computing resources to run the simulations were provided by a Cleveland State University Faculty Research and Development award.

Financial support. This research has been supported by the U.S. Department of Energy, Biological and Environmental Research (grant nos. DE-SC0017999 and DE-SC0019124).

Review statement. This paper was edited by Timothy Garrett and reviewed by two anonymous referees.

\section{References}

Abma, D., Heus, T., and Mellado, J. P.: Direct Numerical Simulation of Evaporative Cooling at the Lateral Boundary of Shallow Cumulus Clouds, J. Atmos. Sci., 70, 2088-2102, https://doi.org/10.1175/jas-d-12-0230.1, 2013.

Ansmann, A., Fruntke, J., and Engelmann, R.: Updraft and downdraft characterization with Doppler lidar: cloud-free versus cumuli-topped mixed layer, Atmos. Chem. Phys., 10, 78457858, https://doi.org/10.5194/acp-10-7845-2010, 2010.

Arakawa, A. and Schubert, W. H.: Interaction of a Cumulus Cloud Ensemble with the Large-Scale Environment, Part I, J. Atmos. Sci., 31, 674-701, https://doi.org/10.1175/15200469(1974)031<0674:ioacce>2.0.co;2, 1974.

ARM: Lasso Bundle Browser, available at: https://adc.arm.gov/ lassobrowser, last access: 26 August 2020a.

ARM: ARM Data archive, available at: https://adc.arm.gov/data/, last access: 26 August 2020b.

Barron, N., Ryan, S. D., and Heus, T.: Reconciling Chord Length Distributions and Area Distributions for Fields of Fractal Cumulus Clouds, Atmosphere, 11, 824, https://doi.org/10.3390/atmos11080824, 2020.

Benner, T. C. and Curry, J. A.: Characteristics of small tropical cumulus clouds and their impact on the environment, J. Geophys. Res.-Atmos., 103, 28753-28767, https://doi.org/10.1029/98jd02579, 1998.

Böing, S. J., Jonker, H. J. J., Siebesma, A. P., and Grabowski, W. W.: Influence of the Subcloud Layer on the Development of a Deep Convective Ensemble, J. Atmos. Sci., 69, 2682-2698, https://doi.org/10.1175/jas-d-11-0317.1, 2012.

Bony, S., Stevens, B., Frierson, D. M. W., Jakob, C., Kageyama, M., Pincus, R., Shepherd, T. G., Sherwood, S. C., Siebesma, A. P., Sobel, A. H., Watanabe, M., and Webb, M. J.: Clouds, circulation and climate sensitivity, Nat. Geosci., 8, 261-268, https://doi.org/10.1038/ngeo2398, 2015.

Brient, F. and Schneider, T.: Constraints on Climate Sensitivity from Space-Based Measurements of Low-Cloud Reflection, J. Climate, 29, 5821-5835, https://doi.org/10.1175/jcli-d-15-0897.1, 2016.

Brooks, M. E., Hogan, R. J., and Illingworth, A. J.: Parameterizing the Difference in Cloud Fraction Defined by Area and by Volume as Observed with Radar and Lidar, J. Atmos. Sci., 62, 2248-2260, https://doi.org/10.1175/jas3467.1, 2005.

Brown, A. R., Cederwall, R. T., Chlond, A., Duynkerke, P. G., Golaz, J. C., Khairoutdinov, M., Lewellen, D. C., Lock, A. P., MacVean, M. K., Moeng, C.-H., Neggers, R. A. J., Siebesma, A. P., and Stevens, B.: Large-eddy simulation of the diurnal cycle of shallow cumulus convection over land, Q. J. Roy. Meteor. Soc., 128, 1075-1093, https://doi.org/10.1256/003590002320373210, 2002.

Dawe, J. T. and Austin, P. H.: Statistical analysis of an LES shallow cumulus cloud ensemble using a cloud tracking algorithm, Atmos. Chem. Phys., 12, 1101-1119, https://doi.org/10.5194/acp12-1101-2012, 2012.

Endo, S., Zhang, D., Vogelmann, A. M., Kollias, P., Lamer, K., Oue, M., Xiao, H., Gustafson, W. I., and Romps, D. M.: Reconciling Differences Between Large-Eddy Simulations and Doppler Lidar Observations of Continental Shallow Cumulus CloudBase Vertical Velocity, Geophys. Res. Lett., 46, 11539-11547, https://doi.org/10.1029/2019gl084893, 2019.

Espy, J. P.: Essays on meteorology. No. IV, J. Franklin I., 22, 239246, https://doi.org/10.1016/S0016-0032(36)91215-2, 1836.

Fast, J. D., Berg, L. K., Feng, Z., Mei, F., Newsom, R., Sakaguchi, K., and Xiao, H.: The Impact of Variable Land-Atmosphere Coupling on Convective Cloud Populations Observed During the 2016 HI-SCALE Field Campaign, J. Adv. Model. Earth Sy., 11, 2629-2654, https://doi.org/10.1029/2019ms001727, 2019.

French, J. R., Vali, G., and Kelly, R. D.: Evolution of small cumulus clouds in Florida: observations of pulsating growth, Atmos. Res., 52, 143-165, https://doi.org/10.1016/s0169-8095(99)00024-1, 1999.

Griewank, P. J., Heus, T., Lareau, N., and Neggers, R. A. J.: Size-dependence in chord characteristics from simulated and observed continental shallow cumulus, Zenodo, https://doi.org/10.5281/zenodo.3731944, 2020.

Gustafson, W., Vogelmann, A., Cheng, X., Endo, S., Johnson, K., Krishna, B., Li, Z., Toto, T., and Xiao., H.: Atmospheric Radiation Measurement (ARM) Research Facility. LASSO Data Bundles, Southern Great Plains Central Facility (C1), ARM Data Archive: Oak Ridge, Tennessee, USA, https://doi.org/10.5439/1342961, 2017.

Gustafson, W. I., Vogelmann, A. M., Cheng, X., Dumas, K. K., Endo, S., Johnson, K. L., Krishna, B., Li, Z., Toto, T., and Xiao, H.: Description of the LASSO Data Bundles Product, Tech. rep., Department of Energy's Office of Scientific and Technical Information, https://doi.org/10.2172/1469590, 2018.

Gustafson, W. I., Vogelmann, A. M., Li, Z., Cheng, X., Dumas, K. K., Endo, S., Johnson, K. L., Krishna, B., Toto, T., and Xiao, H.: The Large-Eddy Simulation (LES) Atmospheric Radiation Measurement (ARM) Symbiotic Simulation and Observation (LASSO) Activity for Continental Shallow Convection, B. Am. 
Meteorol. Soc., 101, E462-E479, https://doi.org/10.1175/bamsd-19-0065.1, 2020.

Hagos, S., Feng, Z., Plant, R. S., Houze, R. A., and Xiao, H.: A Stochastic Framework for Modeling the Population Dynamics of Convective Clouds, J. Adv. Model. Earth Sy., 10, 448-465, https://doi.org/10.1002/2017ms001214, 2018.

Heus, T. and Jonker, H. J. J.: Subsiding Shells around Shallow Cumulus Clouds, J. Atmos. Sci., 65, 1003-1018, https://doi.org/10.1175/2007jas2322.1, 2008.

Heus, T.: microhh release 1.9.1, available at: https://github. com/microhh/microhh2/releases/tag/1.9.1, last access: $26 \mathrm{Au}$ gust 2020 .

Heus, T. and Seifert, A.: Automated tracking of shallow cumulus clouds in large domain, long duration large eddy simulations, Geosci. Model Dev., 6, 1261-1273, https://doi.org/10.5194/gmd6-1261-2013, 2013.

Hoffmann, F., Siebert, H., Schumacher, J., Riechelmann, T., Katzwinkel, J., Kumar, B., Götzfried, P., and Raasch, S.: Entrainment and mixing at the interface of shallow cumulus clouds: Results from a combination of observations and simulations, Meteorol. Z., 23, 349-368, https://doi.org/10.1127/09412948/2014/0597, 2014.

Illingworth, A. J., Hogan, R. J., O’Connor, E., Bouniol, D., Brooks, M. E., Delanoé, J., Donovan, D. P., Eastment, J. D., Gaussiat, N., Goddard, J. W. F., Haeffelin, M., Baltink, H. K., Krasnov, O. A., Pelon, J., Piriou, J.-M., Protat, A., Russchenberg, H. W. J., Seifert, A., Tompkins, A. M., van Zadelhoff, G.-J., Vinit, F., Willén, U., Wilson, D. R., and Wrench, C. L.: Cloudnet, B. Am. Meteorol. Soc., 88, 883-898, https://doi.org/10.1175/bams-88-6883, 2007.

Jung, E. and Albrecht, B.: Use of Radar Chaff for Studying Circulations in and around Shallow Cumulus Clouds, J. Appl. Meteorol. Clim., 53, 2058-2071, https://doi.org/10.1175/jamc-d-130255.1, 2014.

Katzwinkel, J., Siebert, H., Heus, T., and Shaw, R. A.: Measurements of Turbulent Mixing and Subsiding Shells in Trade Wind Cumuli, J. Atmos. Sci., 71, 2810-2822, https://doi.org/10.1175/jas-d-13-0222.1, 2014.

Kitchen, M. and Caughey, S. J.: Tethered-balloon observations of the structure of small cumulus clouds, Q. J. Roy. Meteor. Soc., 107, 853-874, https://doi.org/10.1002/qj.49710745407, 2007.

Laird, N. F., Ochs, H. T., Rauber, R. M., and Miller, L. J.: Initial Precipitation Formation in Warm Florida Cumulus, J. Atmos. Sci., 57, 3740-3751, https://doi.org/10.1175/15200469(2000)057<3740:ipfiwf>2.0.co;2, 2000.

Lamer, K. and Kollias, P.: Observations of fair-weather cumuli over land: Dynamical factors controlling cloud size and cover, Geophys. Res. Lett., 42, 8693-8701, https://doi.org/10.1002/2015g1064534, 2015.

Lareau, N. P.: Subcloud and Cloud-Base Latent Heat Fluxes during Shallow Cumulus Convection, J. Atmos. Sci., 77, 1081-1100, https://doi.org/10.1175/jas-d-19-0122.1, 2020.

Lareau, N. P., Zhang, Y., and Klein, S. A.: Observed Boundary Layer Controls on Shallow Cumulus at the ARM Southern Great Plains Site, J. Atmos. Sci., 75, 2235-2255, https://doi.org/10.1175/jas-d-17-0244.1, 2018.

Mallaun, C., Giez, A., Mayr, G. J., and Rotach, M. W.: Subsiding shells and the distribution of up- and downdraughts in warm cu- mulus clouds over land, Atmos. Chem. Phys., 19, 9769-9786, https://doi.org/10.5194/acp-19-9769-2019, 2019.

Nair, U. S., Weger, R. C., Kuo, K. S., and Welch, R. M.: Clustering, randomness, and regularity in cloud fields: 5 . The nature of regular cumulus cloud fields, J. Geophys. Res.-Atmos., 103, 11363-11380, https://doi.org/10.1029/98jd00088, 1998.

Nam, C. C. W., Quaas, J., Neggers, R., Drian, C. S.-L., and Isotta, F.: Evaluation of boundary layer cloud parameterizations in the ECHAM5 general circulation model using CALIPSO and CloudSat satellite data, J. Adv. Model. Earth Sy., 6, 300-314, https://doi.org/10.1002/2013ms000277, 2014.

Neggers, R. A. J.: Exploring bin-macrophysics models for moist convective transport and clouds, J. Adv. Model. Earth Sy., 7, 2079-2104, https://doi.org/10.1002/2015ms000502, 2015.

Neggers, R. A. J. and Siebesma, A. P.: Constraining a System of Interacting Parameterizations through Multiple-Parameter Evaluation: Tracing a Compensating Error between Cloud Vertical Structure and Cloud Overlap, J. Climate, 26, 6698-6715, https://doi.org/10.1175/jcli-d-12-00779.1, 2013.

Neggers, R. A. J., Jonker, H. J. J., and Siebesma, A. P.: Size Statistics of Cumulus Cloud Populations in Large-Eddy Simulations, J. Atmos. Sci., 60, 1060-1074, https://doi.org/10.1175/15200469(2003)60<1060:ssoccp>2.0.co;2, 2003.

Neggers, R. A. J., Duynkerke, P. G., and Rodts, S. M. A.: Shallow cumulus convection: A validation of large-eddy simulation against aircraft and Landsat observations, Q. J. Roy. Meteor. Soc., 129, 2671-2696, https://doi.org/10.1256/qj.02.93, 2006.

Neggers, R. A. J., Siebesma, A. P., and Heus, T.: Continuous Single-Column Model Evaluation at a Permanent Meteorological Supersite, B. Am. Meteorol. Soc., 93, 1389-1400, https://doi.org/10.1175/bams-d-11-00162.1, 2012.

Newsom, R.: Doppler Lidar (DLFPT), Atmospheric Radiation Measurement (ARM) User Facility, https://doi.org/10.5439/1025185, 2010.

Olson, J. B., Kenyon, J. S., Angevine, W. A., Brown, J. M., Pagowski, M., and Sušelj, K.: A Description of the MYNNEDMF Scheme and the Coupling to Other Components in WRFARW, https://doi.org/10.25923/N9WM-BE49, 2019.

Park, S.: A Unified Convection Scheme (UNICON), Part I: Formulation, J. Atmos. Sci., 71, 3902-3930, https://doi.org/10.1175/jas-d-13-0233.1, 2014.

Peters, J. M., Nowotarski, C. J., and Mullendore, G. L.: Are Supercells Resistant to Entrainment because of Their Rotation?, J. Atmos. Sci., 77, 1475-1495, https://doi.org/10.1175/JAS-D-190316.1, 2020.

Plank, V. G.: The Size Distribution of Cumulus Clouds in Representative Florida Populations, J. Appl. Meteorol., 8, 46-67, https://doi.org/10.1175/15200450(1969)008<0046:tsdocc>2.0.co;2, 1969.

Raga, G. B., Jensen, J. B., and Baker, M. B.: Characteristics of Cumulus Band Clouds off the Coast of Hawaii, J. Atmos. Sci., 47, 338-356, https://doi.org/10.1175/15200469(1990)047<0338:cocbco>2.0.co;2, 1990.

Rodts, S. M. A., Duynkerke, P. G., and Jonker, H. J. J.: Size Distributions and Dynamical Properties of Shallow Cumulus Clouds from Aircraft Observations and Satellite Data, J. Atmos. Sci., 60, 1895-1912, https://doi.org/10.1175/15200469(2003)060<1895:sdadpo>2.0.co;2, 2003. 
Romps, D. M.: Exact Expression for the Lifting Condensation Level, J. Atmos. Sci., 74, 3891-3900, https://doi.org/10.1175/JAS-D-17-0102.1, 2017.

Rossow, W. B.: Measuring Cloud Properties from Space: A Review, J. Climate, 2, 201-213, https://doi.org/10.1175/15200442(1989)002<0201:mcpfsa>2.0.co;2, 1989.

Sakradzija, M. and Klingebiel, M.: Comparing ground-based observations and a large-eddy simulation of shallow cumuli by isolating the main controlling factors of the mass flux distribution, Q. J. Roy. Meteor. Soc., 146, 254-266, https://doi.org/10.1002/qj.3671, 2020.

Schalkwijk, J., Jonker, H. J. J., Siebesma, A. P., and Bosveld, F. C.: A Year-Long Large-Eddy Simulation of the Weather over Cabauw: An Overview, Mon. Weather Rev., 143, 828-844, https://doi.org/10.1175/mwr-d-14-00293.1, 2015.

Sengupta, S. K., Welch, R. M., Navar, M. S., Berendes, T. A., and Chen, D. W.: Cumulus Cloud Field Morphology and Spatial Patterns Derived from High Spatial Resolution Landsat Imagery, J. Appl. Meteorol., 29, 1245-1267, https://doi.org/10.1175/15200450(1990)029<1245:ccfmas $>2.0 . c 0 ; 2,1990$.

Sherwood, S. C., Bony, S., and Dufresne, J.-L.: Spread in model climate sensitivity traced to atmospheric convective mixing, Nature, 505, 37-42, https://doi.org/10.1038/nature12829, 2014.

Siebert, H., Franke, H., Lehmann, K., Maser, R., Saw, E. W., Schell, D., Shaw, R., and Wendisch, M.: Probing Finescale Dynamics and Microphysics of Clouds with HelicopterBorne Measurements, B. Am. Meteorol. Soc., 87, 1727-1738, https://doi.org/10.1175/BAMS-87-12-1727, 2006.

Siebesma, A. P., Bretherton, C. S., Brown, A., Chlond, A., Cuxart, J., Duynkerke, P. G., Jiang, H. L., Khairoutdinov, M., Lewellen, D., Moeng, C. H., Sanchez, E., Stevens, B., and Stevens, D. E.: A large eddy simulation intercomparison study of shallow cumulus convection, J. Atmos. Sci., 60, 1201-1219, https://doi.org/10.1175/1520-0469(2003)60, 2003.

Simpson, J. and Wiggert, V.: Models odf precipitating cumulus towers, Mon. Weather Rev., 97, 471-489, https://doi.org/10.1175/15200493(1969)097<0471:mopct>2.3.co;2, 1969.

Tucker, S. C., Senff, C. J., Weickmann, A. M., Brewer, W. A., Banta, R. M., Sandberg, S. P., Law, D. C., and Hardesty, R. M.: Doppler Lidar Estimation of Mixing Height Using Turbulence, Shear, and Aerosol Profiles, J. Atmos. Ocean. Tech., 26, 673688, https://doi.org/10.1175/2008jtecha1157.1, 2009.

Turner, D. D., Ferrare, R. A., Wulfmeyer, V., and Scarino, A. J.: Aircraft Evaluation of Ground-Based Raman Lidar Water Vapor Turbulence Profiles in Convective Mixed Layers, J. Atmos. Ocean. Tech., 31, 1078-1088, https://doi.org/10.1175/jtech-d13-00075.1, 2014a.

Turner, D. D., Wulfmeyer, V., Berg, L. K., and Schween, J. H.: Water vapor turbulence profiles in stationary continental convective mixed layers, J. Geophys. Res.-Atmos., 119, 11151-11165, https://doi.org/10.1002/2014jd022202, 2014b.

Turner, J. S.: The "starting plume" in neutral surroundings, J. Fluid Mech., 13, 356-368, https://doi.org/10.1017/s0022112062000762, 1962. van Heerwaarden, C. C., van Stratum, B. J. H., Heus, T., Gibbs, J. A., Fedorovich, E., and Mellado, J. P.: MicroHH 1.0: a computational fluid dynamics code for direct numerical simulation and large-eddy simulation of atmospheric boundary layer flows, Geosci. Model Dev., 10, 3145-3165, https://doi.org/10.5194/gmd-10-3145-2017, 2017.

van Laar, T. W., Schemann, V., and Neggers, R. A. J.: Investigating the Diurnal Evolution of the Cloud Size Distribution of Continental Cumulus Convection Using Multiday LES, J. Atmos. Sci., 76, 729-747, https://doi.org/10.1175/jas-d-18-0084.1, 2019.

Wang, Y. and Geerts, B.: Humidity variations across the edge of trade wind cumuli: Observations and dynamical implications, Atmos. Res., 97, 144-156, https://doi.org/10.1016/j.atmosres.2010.03.017, 2010.

Wang, Y. and Geerts, B.: Observations of detrainment signatures from non-precipitating orographic cumulus clouds, Atmos. Res., 99, 302-324, https://doi.org/10.1016/j.atmosres.2010.10.023, 2011.

Warner, J.: On Steady-State One-Dimensional Models of Cumulus Convection, J. Atmos. Sci., 27, 1035-1040, https://doi.org/10.1175/15200469(1970)027<1035:ossodm>2.0.co;2, 1970a.

Warner, J.: The Microstructure of Cumulus Cloud. Part III. The Nature of the Updraft, J. Atmos. Sci., 27, 682-688, https://doi.org/10.1175/15200469(1970)027<0682:TMOCCP>2.0.CO;2, 1970b.

Wood, R. and Field, P. R.: The Distribution of Cloud Horizontal Sizes, J. Climate, 24, 4800-4816, https://doi.org/10.1175/2011jcli4056.1, 2011.

Wulfmeyer, V., Pal, S., Turner, D. D., and Wagner, E.: Can Water Vapour Raman Lidar Resolve Profiles of Turbulent Variables in the Convective Boundary Layer?, Bound.-Lay. Meteorol., 136, 253-284, https://doi.org/10.1007/s10546-010-9494-z, 2010.

Yano, J.-I.: Basic convective element: bubble or plume? A historical review, Atmospheric Chemistry and Physics, 14, 7019-7030, https://doi.org/10.5194/acp-14-7019-2014, 2014.

Yuan, T.: Cloud macroscopic organization: order emerging from randomness, Atmos. Chem. Phys., 11, 7483-7490, https://doi.org/10.5194/acp-11-7483-2011, 2011.

Zhang, Y., Klein, S. A., Fan, J., Chandra, A. S., Kollias, P., Xie, S., and Tang, S.: Large-Eddy Simulation of Shallow Cumulus over Land: A Composite Case Based on ARM Long-Term Observations at Its Southern Great Plains Site, J. Atmos. Sci., 74, 3229-3251, https://doi.org/10.1175/jas-d-16-0317.1, 2017.

Zhao, G. and Girolamo, L. D.: Statistics on the macrophysical properties of trade wind cumuli over the tropical western Atlantic, J. Geophys. Res.-Atmos., 112, D10204, https://doi.org/10.1029/2006jd007371, 2007.

Zhao, M. and Austin, P. H.: Life Cycle of Numerically Simulated Shallow Cumulus Clouds. Part II: Mixing Dynamics, J. Atmos. Sci., 62, 1291-1310, https://doi.org/10.1175/jas3415.1, 2005. 